\title{
Statistical Comparison of the Baseline Mechanical Properties of NBG-18 and PCEA Graphite
}

M.C. Carroll and D.T. Rohrbaugh

August 2013

The INL is a

U.S. Department of Energy

National Laboratory

operated by

Battelle Energy Alliance

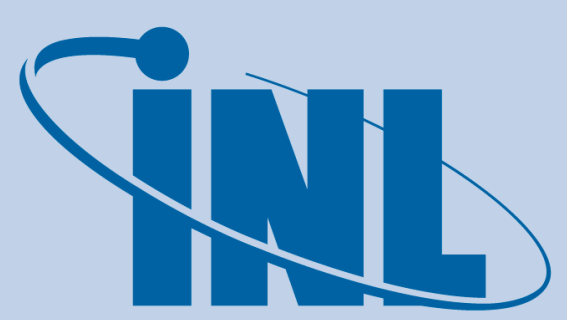

Idaho National Laboratory 


\section{DISCLAIMER}

This information was prepared as an account of work sponsored by an agency of the U.S. Government. Neither the U.S. Government nor any agency thereof, nor any of their employees, makes any warranty, expressed or implied, or assumes any legal liability or responsibility for the accuracy, completeness, or usefulness, of any information, apparatus, product, or process disclosed, or represents that its use would not infringe privately owned rights. References herein to any specific commercial product, process, or service by trade name, trade mark, manufacturer, or otherwise, does not necessarily constitute or imply its endorsement, recommendation, or favoring by the U.S. Government or any agency thereof. The views and opinions of authors expressed herein do not necessarily state or reflect those of the U.S. Government or any agency thereof. 


\title{
Statistical Comparison of the Baseline Mechanical Properties of NBG-18 and PCEA Graphite
}

\author{
M.C. Carroll and D.T. Rohrbaugh
}

August 2013

\begin{abstract}
Idaho National Laboratory
VHTR Program

Idaho Falls, Idaho 83415
\end{abstract}

http://www.inl.gov

Prepared for the

U.S. Department of Energy

Office of Nuclear Energy

Under DOE Idaho Operations Office

Contract DE-AC07-05ID14517 



\section{VHTR Program}

\section{Statistical Comparison of the Baseline Mechanical Properties of NBG-18 and PCEA Graphite}

INL/EXT-13-30011

August 2013

Approved by:

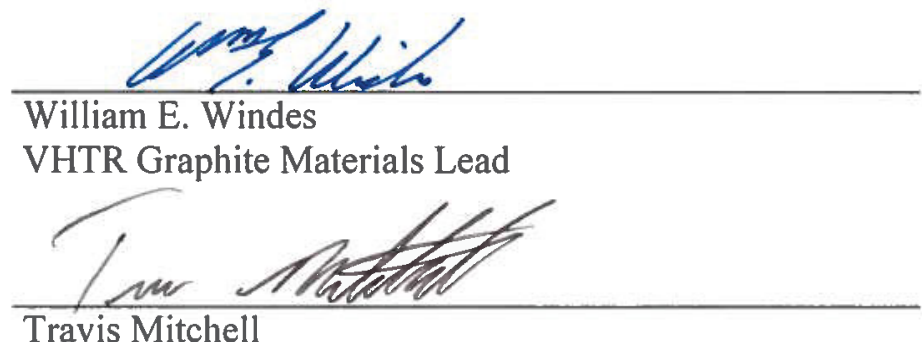

Travis Mitchell

30 Angurt 20/3

VHTR TDO Program Manager

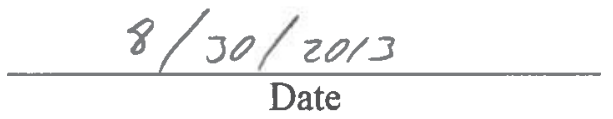





\section{ABSTRACT}

High-purity graphite is the core structural material of choice in the Very High Temperature Reactor (VHTR) design, a graphite-moderated, helium-cooled configuration that is capable of producing thermal energy for power generation as well as process heat for industrial applications that require temperatures higher than the outlet temperatures of present nuclear reactors.

The Baseline Graphite Characterization Program is endeavoring to minimize the conservative estimates of as-manufactured mechanical and physical properties in nuclear-grade graphites by providing comprehensive data that captures the level of variation in measured values. In addition to providing a comprehensive comparison between these values in different graphite grades, the program is also carefully tracking individual specimen source, position, and orientation information in order to provide comparisons both in specific properties and in the associated variability between different lots, different billets, and different positions from within a single billet.

This report is a preliminary comparison between the two grades of graphite that were initially favored in the two main VHTR designs. NBG-18, a medium-grain pitch coke graphite from SGL from which billets are formed via vibration molding, was the favored structural material in the pebble-bed configuration. PCEA, a smaller grain, petroleum coke graphite from GrafTech formed via an extrusion process, was the favored grade for the prismatic layout.

An analysis of the comparison between these two grades will include not only the differences in fundamental and statistically-significant individual strength levels, but also the differences in variability in properties within each of the grades that will ultimately provide the basis for the prediction of inservice performance. The comparative performance of the different types of nuclear grade graphites will continue to evolve as thousands more specimens are fully characterized from the numerous grades of graphite being evaluated. 


\section{CONTENTS}

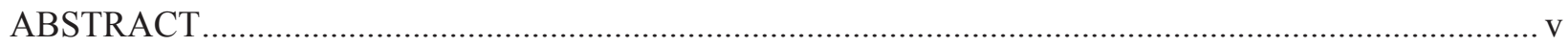

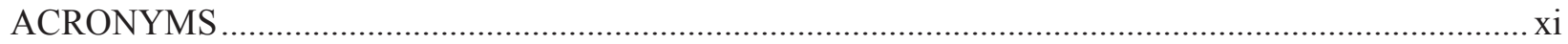

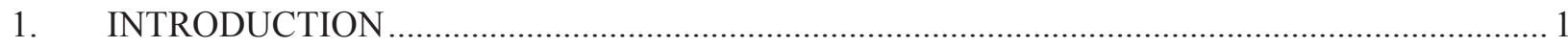

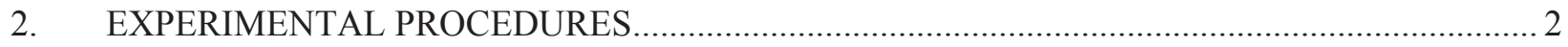

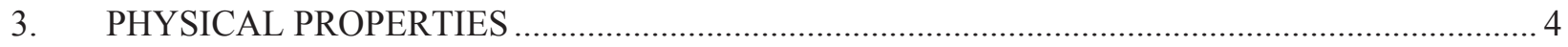

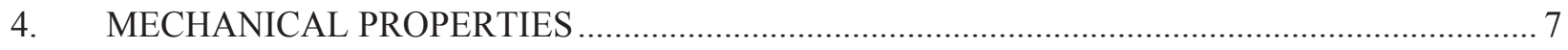

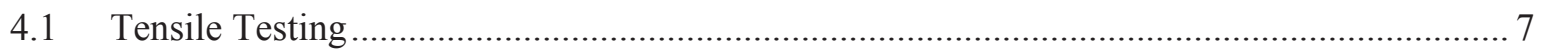

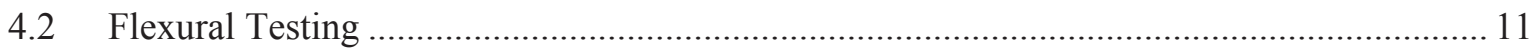

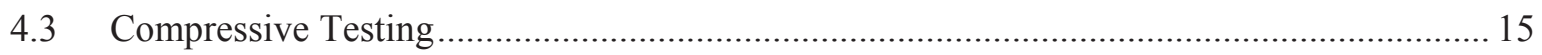

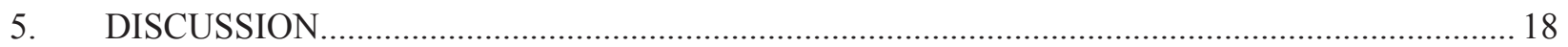

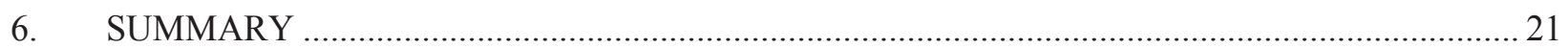

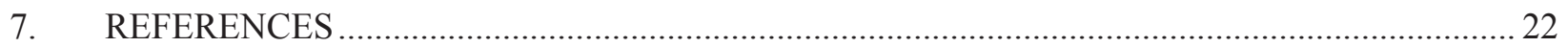

\section{FIGURES}

Figure 1. Examples of the ASTM-based configurations for (a) tensile, (b) flexural, and (c) compression testing of nuclear-grade graphite.

Figure 2. Rectangular billets of vibration-molded NBG-18 are sectioned into 7 slabs and quartered into sub-blocks, from which three orientations of test specimens can be extracted.

Figure 3. Cylindrical extruded billets of PCEA graphite are sectioned into 7 slabs along the z-axis and quartered into sub-wedges, from which test specimens are extracted in parallel, transverse, and radial orientations.

Figure 4. A 3D representation of all of the density data that has been collected to date for the vibration molded (left) and extruded (right) grades of graphite. Distinct density gradients exist in the extruded graphite that are not as readily apparent in the vibrationmolded grade.

Figure 5. A qualitative evaluation of the density distribution for each grade is made through a Weibull probability plot. The vibration-molded (VM) graphite shows higher mean strength values, as shown by the characteristic density value (scale parameter) of 1.851 $\mathrm{g} / \mathrm{cc}$ vs. $1.816 \mathrm{~g} / \mathrm{cc}$ in the extruded grade. The VM graphite is also better represented by a Weibull curve fit, and shows less overall variation as evidenced by the steeper slope (shape parameter).

Figure 6. Dynamic Young's modulus (left) and shear modulus (right) based on resonant frequencies of vibration measured in flexural test specimens. The vibration-molded grade exhibits higher stiffness in both vibration modes throughout the distribution of values. 
Figure 7. Dynamic Young's modulus comparison between the individual billets of vibrationmolded graphite (left) and extruded graphite (right). The vibration-molded graphite (plotted on a much tighter $\mathrm{x}$-axis scale) exhibits considerably less modulus variability

Figure 8. Young's modulus (left) and shear modulus (right) comparisons between the grades reveal similar higher stiffness values and a tighter distribution for the vibration-molded grade, with the individual data points (both modulus types were taken from each specimen measured) indicating similar values from each specimen based upon the similar distribution shapes.

Figure 9. A comparison of the modulus of elasticity (Young's modulus) for vibration molded (left) and extruded (right) grades of graphite using two different measurement techniques. The modulus by sonic velocity exhibits considerably less variability and a lower incidence of low-value outliers.

Figure 10. A qualitative 3D representation of the distribution of tensile strength values in vibration molded (left) and extruded (right) graphite grades. The gradient in the extruded graphite is reflective of the same pattern in the density distributions of Figure 4.

Figure 11. Weibull distributions of the tensile strengths measured in each grade show a higher characteristic value of $20.40 \mathrm{MPa}$ for the vibration-molded grade vs. $18.59 \mathrm{MPa}$ for the extruded grade, despite the upper values for the extruded grade being higher. The overall distribution shows less variability at a higher overall mean value for the vibration-molded grade.

Figure 12. The distribution in tensile values from end to end in the billet (represented by grouping the data as Slabs 1-7) indicates that the trend in decreasing value from "top" to "bottom" is consistent to varying degrees through each of the three individual billets (bottom 3 boxplots).

Figure 13. The variability between orientations is more pronounced in the extruded grade, with the radial orientation exhibiting the highest strength. The distribution of values is much closer in the vibration-molded grade, with the highest characteristic value for strength being shown by the orientation commonly regarded as the weakest - the against-grain orientation.

Figure 14. As with the physical property measurements, the vibration-molded grade exhibits higher stiffness across the distribution of values (top). The comparison of test techniques to resulting values further illustrates the effects of test method on the parameter being evaluated; the modulus by stress/strain measurements is consistently lower than the non-destructive techniques (resonant frequency and sonic velocity) presented in the previous section.

Figure 15. 3D representation of flexural strength results for each of the two grades shows a more homogeneous distribution for the vibration molded graphite than for the extruded grade, which further reflects the gradients seen in the density distribution of Figure 4.

Figure 16. The flexural strength values are similar in distribution to the tensile test results, exhibiting a crossover region at the higher values for the extruded graphite. Unlike the tensile results, the characteristic value for flexure strength is higher for the extruded grade despite the larger range of variability in that grade, particularly at the low strength values.

Figure 17. Flexural strength between billets of the same grade for vibration-molded (left) and extruded (right) graphite. 
Figure 18. The end-to-end variability and general spread in data is clear in the extruded grade (top left), with values decreasing by z-axis length via slab number groupings. The vibrationmolded grade (top right) exhibits a similar mean value throughout. The decreasing trend in mean values is confirmed by the stacked ANOVA (right).

Figure 19. Flexural strength by orientation in each of the grades exhibits similar results to the tensile data - in the vibration molded graphite, the results in each of the orientations is similar, with the against-grain orientation showing the highest strength value for the distribution. The radial orientation is the strongest in the extruded grades (right), while the parallel orientation is weakest.

Figure 20. The 3D representation of the compressive strength values for each of the two grades shows a similar qualitative distribution to that seen in the previously evaluated properties, with the compressive strength exhibiting an end-to-end and center-outside gradient..

Figure 21. Compressive strength values for the billets tested show greater variability and a lower overall strength distribution in the extruded grade.

Figure 22. Compressive strength distributions for the vibration-molded billets (left) and extruded billets (right) indicate a narrower range of variability for individual vibration-molded graphite than for extruded.

Figure 23. The compressive strength distributions by orientation overlap are consistent at the upper values for the vibration-molded graphite (left), with higher variability and lower overall values for the against-grain orientation in the lower range. The extruded grade (right) shows the same pattern for mechanical strength as seen previously - the radial orientation is the most consistent and strongest orientation, while the parallel orientation has the weakest distribution of values.

Figure 24. The end-to-end trend variation in the extruded grade shows the same trend in decreasing values by slab number (left) with confirmation of the variability in mean values shown by an ANOVA evaluation (right)......

Figure 25. Boxplots confirm the increasing trend in strength values in the radial orientation from centerline to the outer edge of the extruded grade seen qualitatively in the $3 \mathrm{D}$ plot of Figure 20 .

Figure 26. The 3D plot (left) high strength distribution in the upper layer of the extruded billet and a lower strength region in the bottom center section of the billet. These regions can be quantified by extracting data from the subgroups that compose those specific positions in each of the billets (right).

Figure 27. A boxplot of the two regions (left) shows the difference by directed subgroup of the variation from high strength to low strength regions in the extruded graphite. The specific distribution of strength values from each of those regions (right) provides confirmation of the variation and characteristic value levels.

Figure 28. Distribution of the largest disparate flaws based upon the resolution of individual extraction and handling of specimen coupons. 


\section{ACRONYMS}

$\begin{array}{ll}\text { ANOVA } & \text { analysis of variance } \\ \text { ASTM } & \text { American Society for Testing and Materials } \\ \text { EX } & \text { extruded graphite } \\ \text { VHTR } & \text { Very High Temperature Reactor } \\ \text { VM } & \text { vibration-molded graphite }\end{array}$




\section{Statistical Comparison of the Baseline Mechanical Properties of NBG-18 and PCEA Graphite}

\section{INTRODUCTION}

High-purity graphite is the core structural material of choice in the conceptual design for the Very High Temperature Reactor (VHTR), a graphite-moderated, helium-cooled configuration that is capable of producing thermal energy for power generation as well as process heat for industrial applications that require temperatures higher than the outlet temperatures of present nuclear reactors. While nuclear-grade graphite is an ideal material for this design based upon its extremely high temperature capabilities coupled with an optimum combination of thermal stability, machinability, and low cost, the quasi-brittle mechanical properties of graphite have been shown to exhibit a relatively large amount of scatter in measured strength levels. ${ }^{1}$ The mechanical response of nuclear graphites are dependent upon the inherent defect structure, composed of boundaries between filler particles, pores, voids, inclusions, and cracks that are present at all practical length scales. ${ }^{2}$ The measured mechanical properties in various graphite specimens will be strongly based upon the size distribution of these defects and their relative orientation with respect to the stress axis.

The Baseline Graphite Characterization Program is endeavoring to minimize the conservative estimates of as-manufactured mechanical and physical properties in nuclear-grade graphites by providing comprehensive data that captures the level of variation in measured values. In addition to providing a comprehensive comparison between these values in different graphite grades, the program is also carefully tracking individual specimen source, position, and orientation information in order to provide comparisons in properties between different lots, different billets, and different positions from within a single billet. This analysis includes not only mean values, but also a full analysis of the inherent variability in those properties in order to provide a full accounting of the expected critical properties of interest for nuclear applications.

This report is a preliminary comparison between the two grades of graphite that were initially favored in two of the preliminary VHTR designs. ${ }^{3} \mathrm{NBG}-18$, a medium-grain pitch coke graphite from SGL from which billets are formed via vibration molding, was the favored structural material in the pebble-bed configuration. PCEA, a smaller grain, petroleum coke graphite from GrafTech formed via an extrusion process, was the favored grade for the prismatic layout. These were the first two grades obtained by the program as orders from full lots, and are the first grades to have sufficient data to begin to provide gradeand billet-based comparisons. As the program has evolved, the range of potential graphites produced internationally that are part of this extensive materials qualification process has expanded to include NBG-17 from SGL, 2114 from Mersen, and IG-110 from Toyo Tanso. ${ }^{4}$ Accordingly, those grades of graphite have been or are in the process of being procured and will be fully integrated into the overall characterization program and properties database. This particular report will focus on the graphite from the aforementioned two initial grades, three full-size billets of NBG-18 and three billets of PCEA, that have been comprehensively tested thus far. It will include data obtained both from non-destructive physical properties measurements and the strength property data gathered from the testing to failure of 3,142 mechanical test specimens. 
An analysis of the comparison between these two grades will include not only the differences in fundamental and statistically-significant individual strength levels, but also the differences in variability in properties within each of the grades that will ultimately provide the basis for the prediction of inservice performance. The comparative performance of the different types of nuclear grade graphites will continue to evolve as thousands more specimens are fully characterized from the numerous grades of graphite being evaluated.

\section{EXPERIMENTAL PROCEDURES}

The physical and mechanical properties being reported are based upon a systematic evaluation of specimens machined to the specific guidelines of the published standards from American Society for Testing and Materials (ASTM) International. Tensile testing (Figure 1a) is carried out via ASTM C749$08,{ }^{5}$ flexural testing (Figure 1b) is carried out via ASTM C651-10, ${ }^{6}$ and compressive testing (Figure 1c) is carried out via ASTM C695-91. ${ }^{7}$ The relatively simple shapes

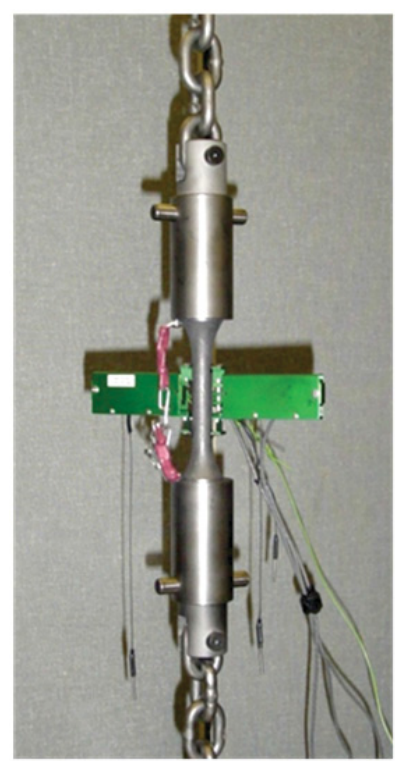

(a)

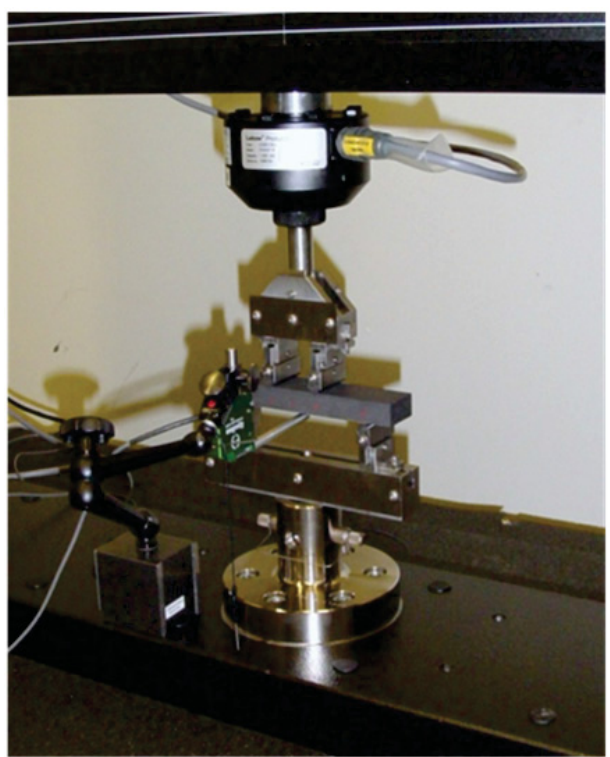

(b)

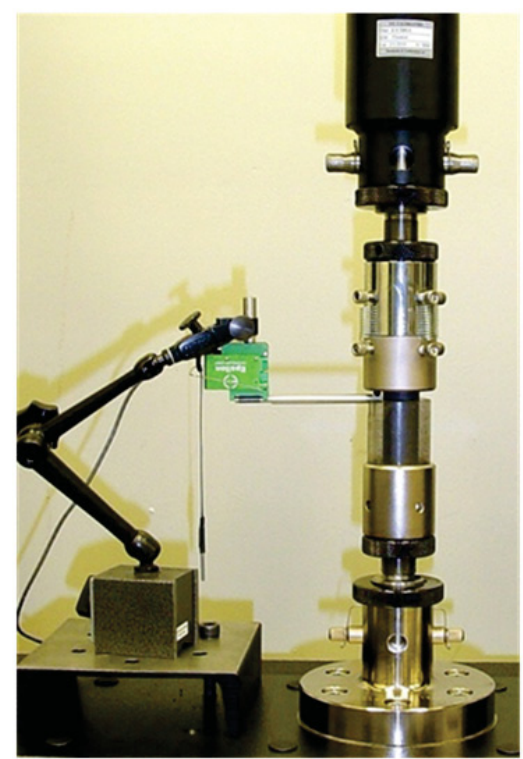

(c)

Figure 1. Examples of the ASTM-based configurations for (a) tensile, (b) flexural, and (c) compression testing of nuclear-grade graphite.

of flexural (rectangular bars) and compressive specimens (right circular cylinders) make them ideal for the non-destructive evaluation of elastic material constants, such as dynamic Young's modulus and shear modulus values, which can be obtained through measurements of resonant frequency (ASTM C747-93) ${ }^{8}$ and sonic velocity (ASTM C769-09). ${ }^{9}$ Additionally, the respective geometries of those sample types render accurate geometry/volume measurements relatively straightforward, which allows for the reporting of material density per ASTM C559-90. ${ }^{10}$ These evaluations are performed on specimens prior to mechanical testing, allowing the individual position information of each specimen to be utilized to describe multiple properties from within a single billet. 
The goals of this program necessitate the accurate tracking of individual specimen source, position, and orientation information, each of which is recorded and embedded in the applicable test files. PCEA and NBG-18 graphites were sectioned and test specimens were extracted in a manner that reflects not only the geometry of the as-manufactured billet, but also the forming technique used to compact the carbonaceous filler and binder into shape prior to graphitization. NBG-18 was sectioned into 7 slabs or

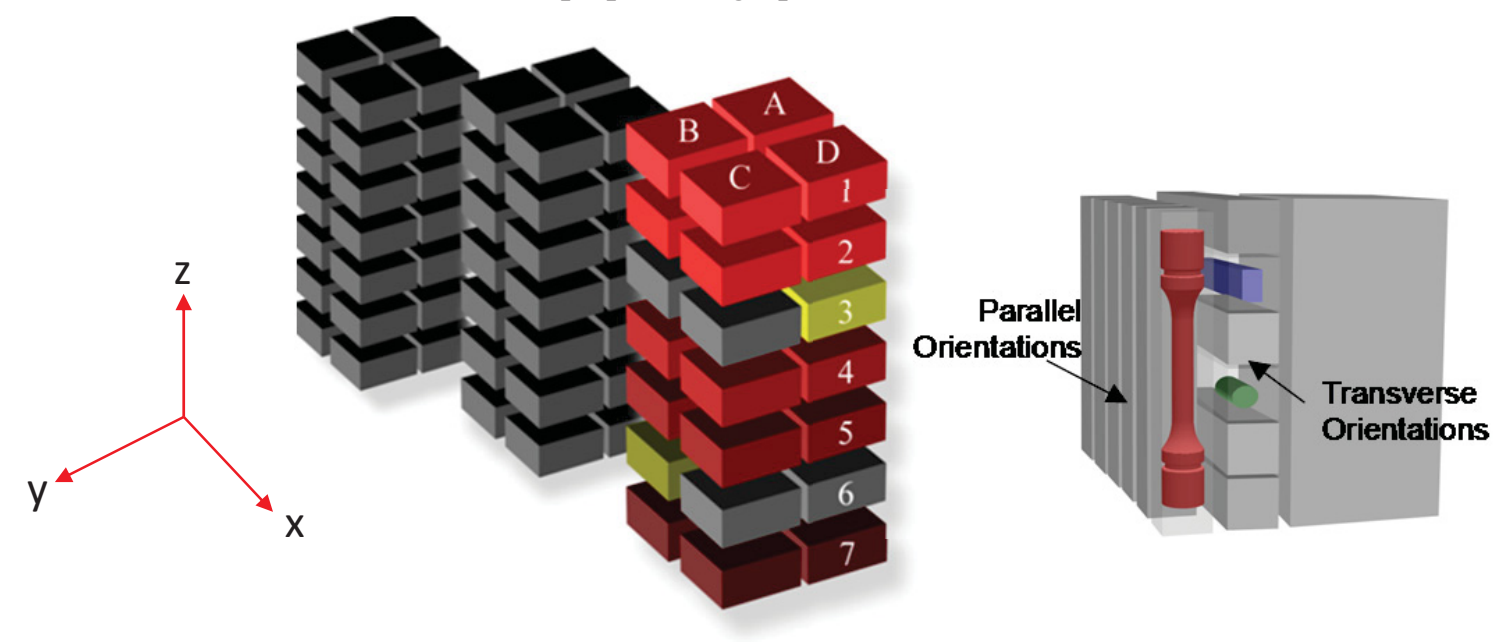

Figure 2. Rectangular billets of vibration-molded NBG-18 are sectioned into 7 slabs and quartered into sub-blocks, from which three orientations of test specimens can be extracted.

layers perpendicular to the long axis, from which individual test specimens were extracted from numerous positions and layers (Figure 2) based on a parallel (long axis) orientation and two orthogonal transverse (with grain and against grain) orientations. The extrusion process for PCEA resulted in a geometry that was similarly sectioned into 7 layers that yielded ideal parallel and transverse orientations, but also added an orientation (radial) that is tangential to the outer radius at varying distances from the billet centerline (Figure 3).
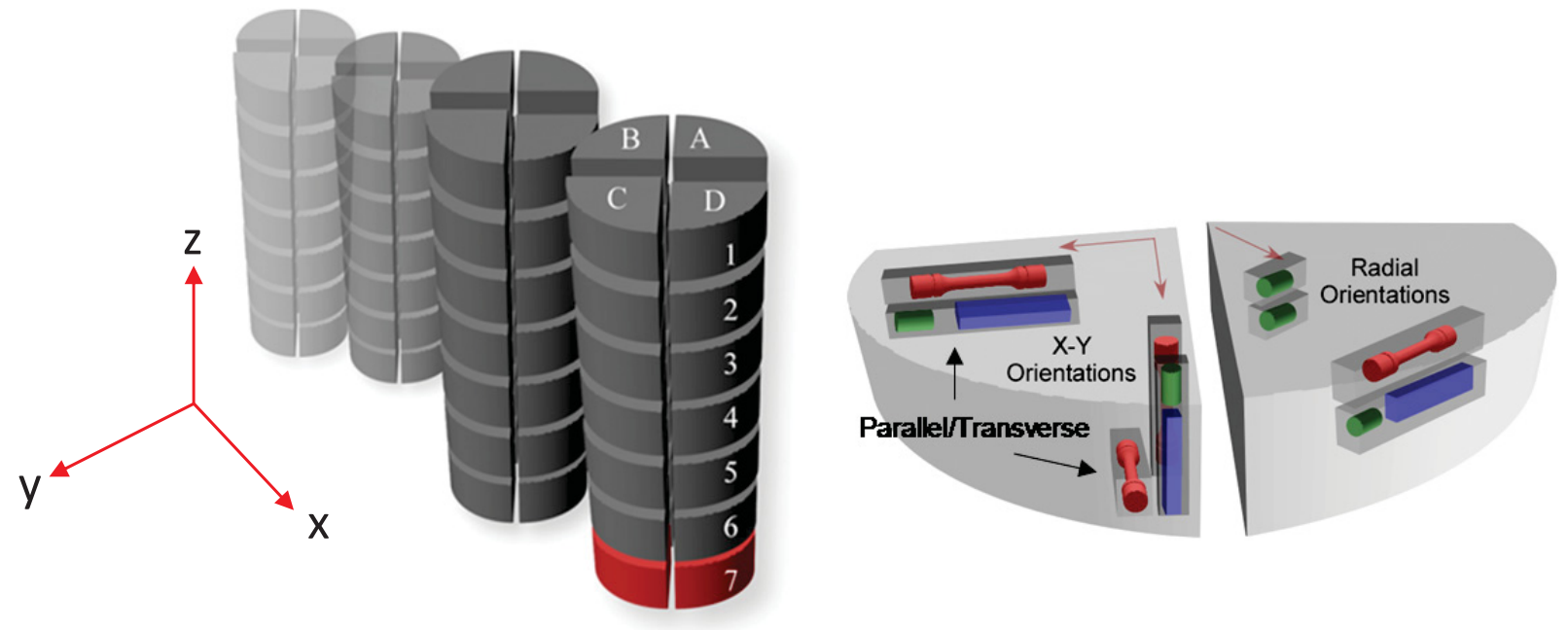

Figure 3. Cylindrical extruded billets of PCEA graphite are sectioned into 7 slabs along the z-axis and quartered into sub-wedges, from which test specimens are extracted in parallel, transverse, and radial orientations. 


\section{PHYSICAL PROPERTIES}

A qualitative comparison between whole grades is most readily observed through a $3 \mathrm{D}$ representation of the original billet that reflects property data based upon the measured value (color) and actual position within the billet. Figure 4 shows the density values measured in both NBG-18 and PCEA, from which it
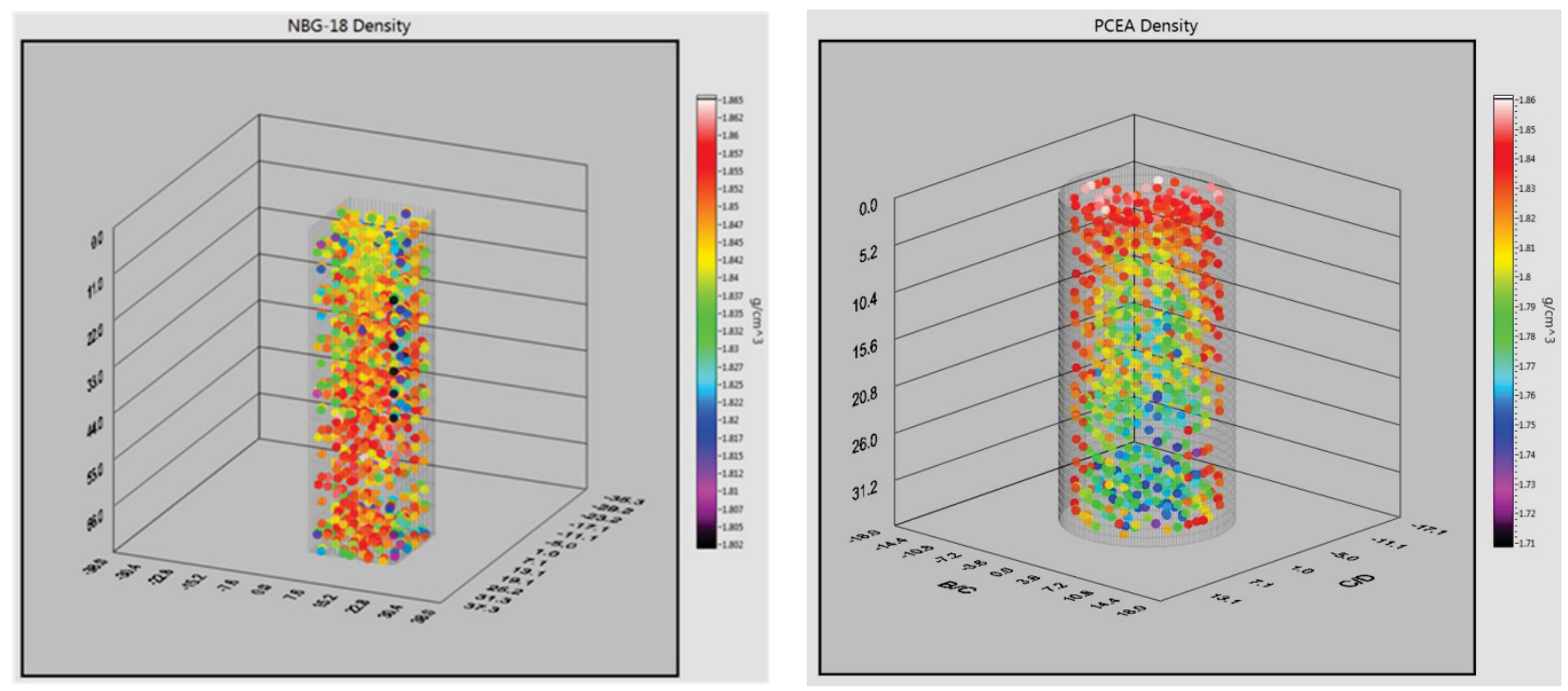

Figure 4. A 3D representation of all of the density data that has been collected to date for the vibration molded (left) and extruded (right) grades of graphite. Distinct density gradients exist in the extruded graphite that are not as readily apparent in the vibration-molded grade.

is clear that the density of the extruded graphite has an end-to-end variability based upon the relative range of values measured as well as a slight inside-outside gradient with higher density values nearer the billet surface. A more quantitative representation of the range of values is made through a probability distribution, as is shown in Figure 5, which utilizes an appropriate curve fit to determine a characteristic value based upon the distribution as well as a quantitative measurement of the shape parameter, or degree of variability in the overall population, based on the slope of the distribution. In the case of density measurements on flexural and compressive mechanical test specimens, the vibration molded graphite exhibits less overall variability in the distribution (and therefore greater predictability) based upon the higher slope value than is shown by the extruded graphite distribution. Per ASTM D7219-08, ${ }^{11}$ graphite has a minimum "allowable" density of $1.7 \mathrm{~g} / \mathrm{cc}$ to be considered nuclear-grade, a value that has an integral effect on the core neutronics and predictability of the moderated neutron flux in an operating reactor, and the vast majority of the measured values fall within this range. Outside of the relationship to core power analyses and ultimate operational predictability, density is not considered a performance property. It is, however, a relatively accurate predictor of the relative variation in other physical and mechanical properties, so trends in this same end-to-end variability will be explored in the analysis of other test results. 


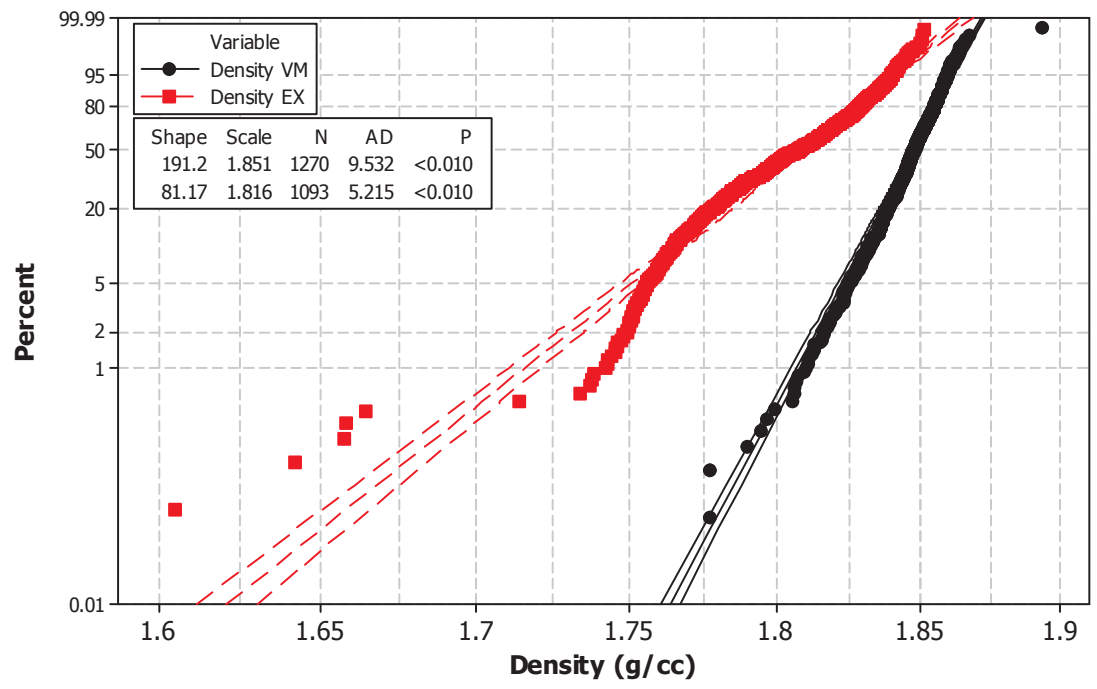

Figure 5. A qualitative evaluation of the density distribution for each grade is made through a Weibull probability plot. The vibration-molded (VM) graphite shows higher mean strength values, as shown by the characteristic density value (scale parameter) of $1.851 \mathrm{~g} / \mathrm{cc}$ vs. $1.816 \mathrm{~g} / \mathrm{cc}$ in the extruded grade. The VM graphite is also better represented by a Weibull curve fit, and shows less overall variation as evidenced by the steeper slope (shape parameter).

Figure 6 is a comparison between the elastic and shear moduli in NBG-18 and PCEA measured via resonant frequency measurements in flexural bars. Figure 7 shows the variation between values in individual billets of each grade. As can be seen from the figures, the variability in extruded graphite is higher than that seen in the vibration-molded grade.
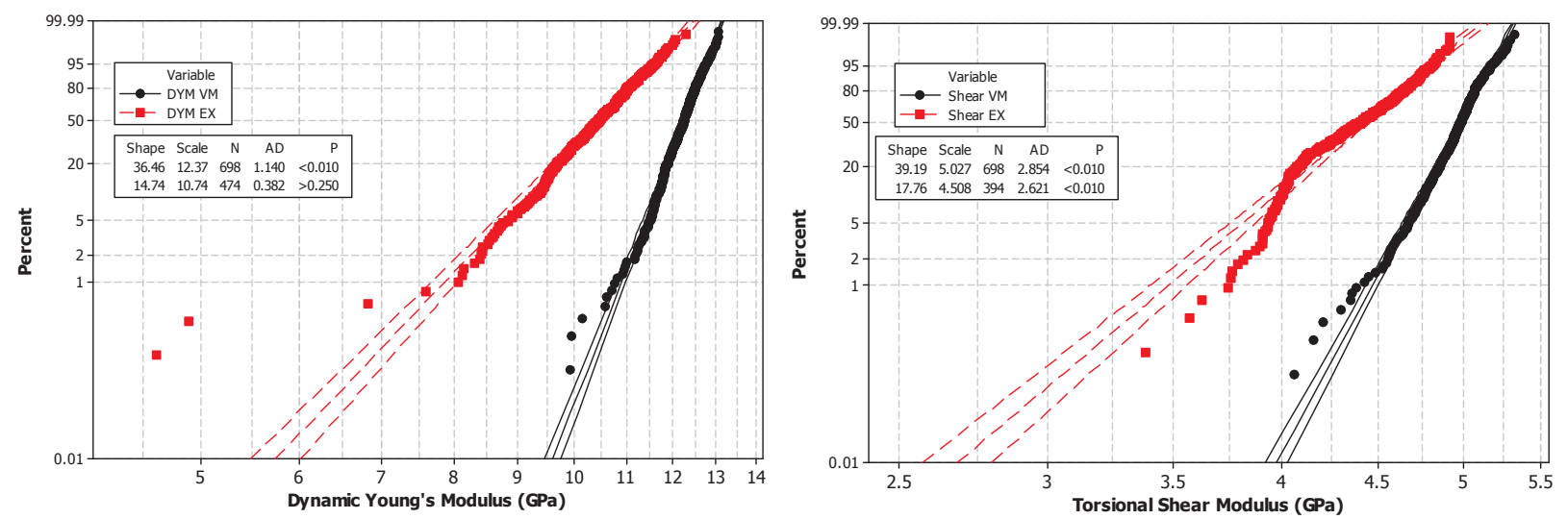

Figure 6. Dynamic Young's modulus (left) and shear modulus (right) based on resonant frequencies of vibration measured in flexural test specimens. The vibration-molded grade exhibits higher stiffness in both vibration modes throughout the distribution of values. 

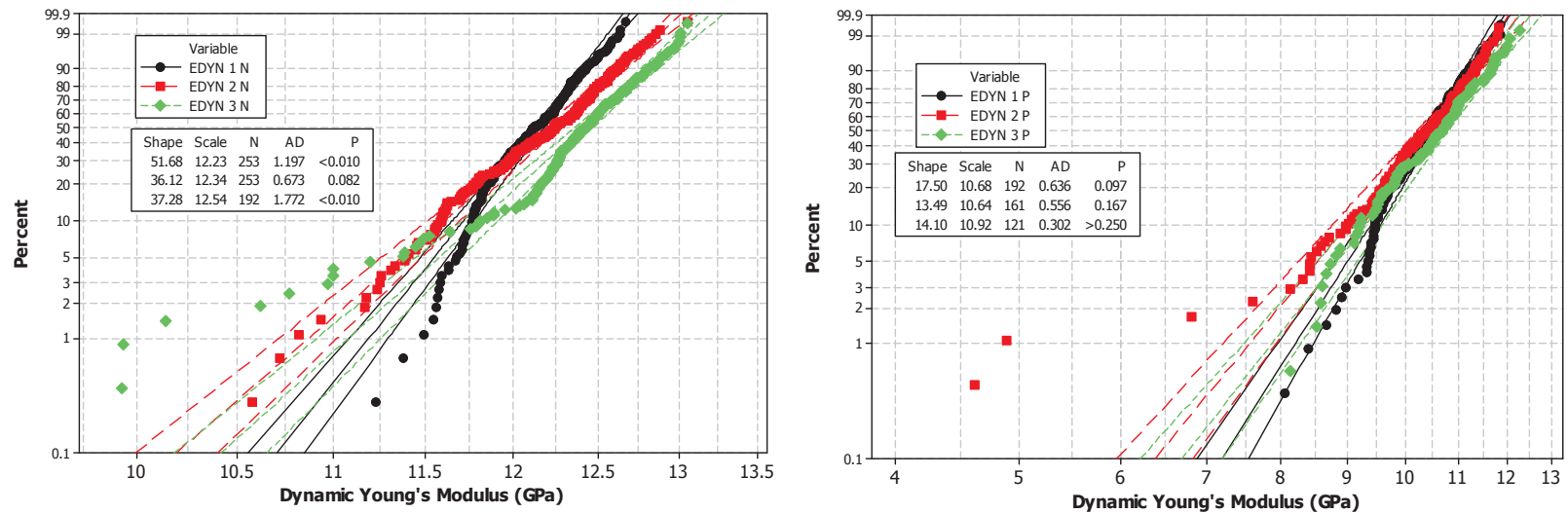

Figure 7. Dynamic Young's modulus comparison between the individual billets of vibration-molded graphite (left) and extruded graphite (right). The vibration-molded graphite (plotted on a much tighter $x$ axis scale) exhibits considerably less modulus variability.

The same elastic constants can be evaluated through other means. In this work, sonic velocity measurements are used to evaluate those elastic constants using compression specimens (Figure 8). The data shows a similar trend in the difference between the grades, with the vibration-molded graphite exhibiting higher stiffness and an overall lower variability in the distribution of values.
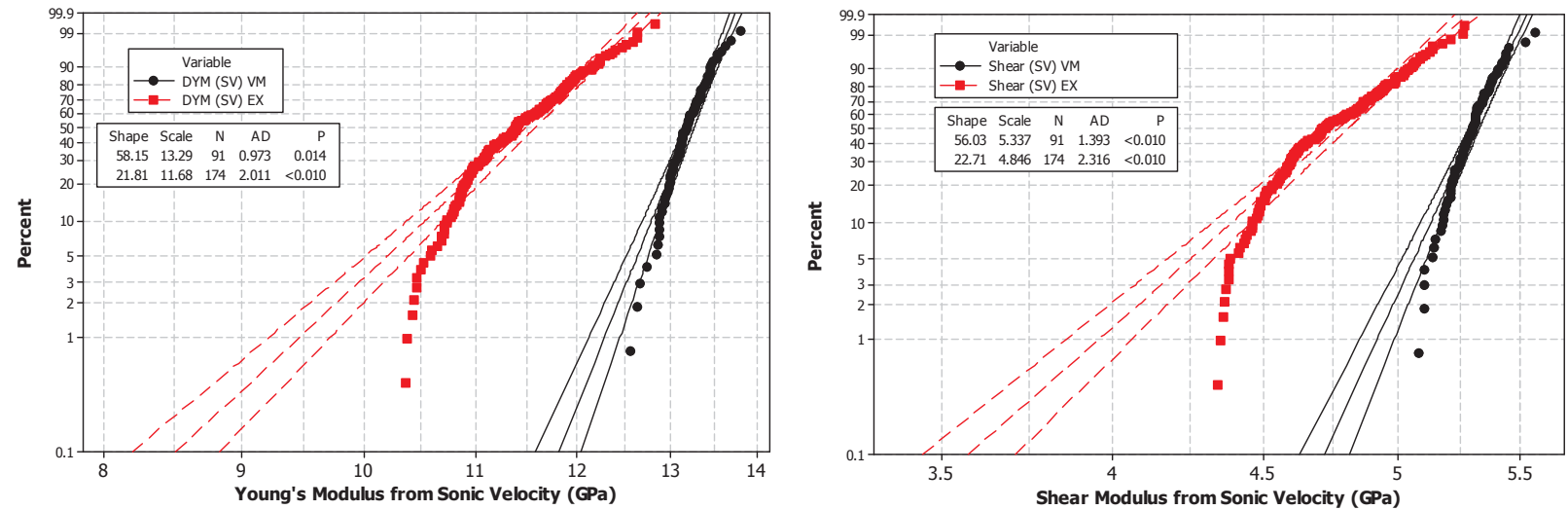

Figure 8. Young's modulus (left) and shear modulus (right) comparisons between the grades reveal similar higher stiffness values and a tighter distribution for the vibration-molded grade, with the individual data points (both modulus types were taken from each specimen measured) indicating similar values from each specimen based upon the similar distribution shapes.

Another key characteristic in evaluating the inherent flaw distribution in the material is the comparison of these elastic constants, presumably similar, that were obtained using different techniques. As shown in Figure 9, a direct comparison of the calculated Young's modulus based on sonic velocity and resonant frequency measurements for each grade exhibits a different degree of variability. The distributions for both grades exhibit considerably less variability for the modulus of the material based upon sonic velocity measurements, despite the fact that both techniques are measuring the same material parameter. The result is indicative of the effect of flaw and void population on the transmission of 
vibrations that are resonating in the specimen volume vs. the interaction of those same flaws and the associated attenuation of a planar sound wave. The individual techniques can provide additional information as to the character of the flaw and void distribution in nuclear grade graphite through a comparison of the same physical property.
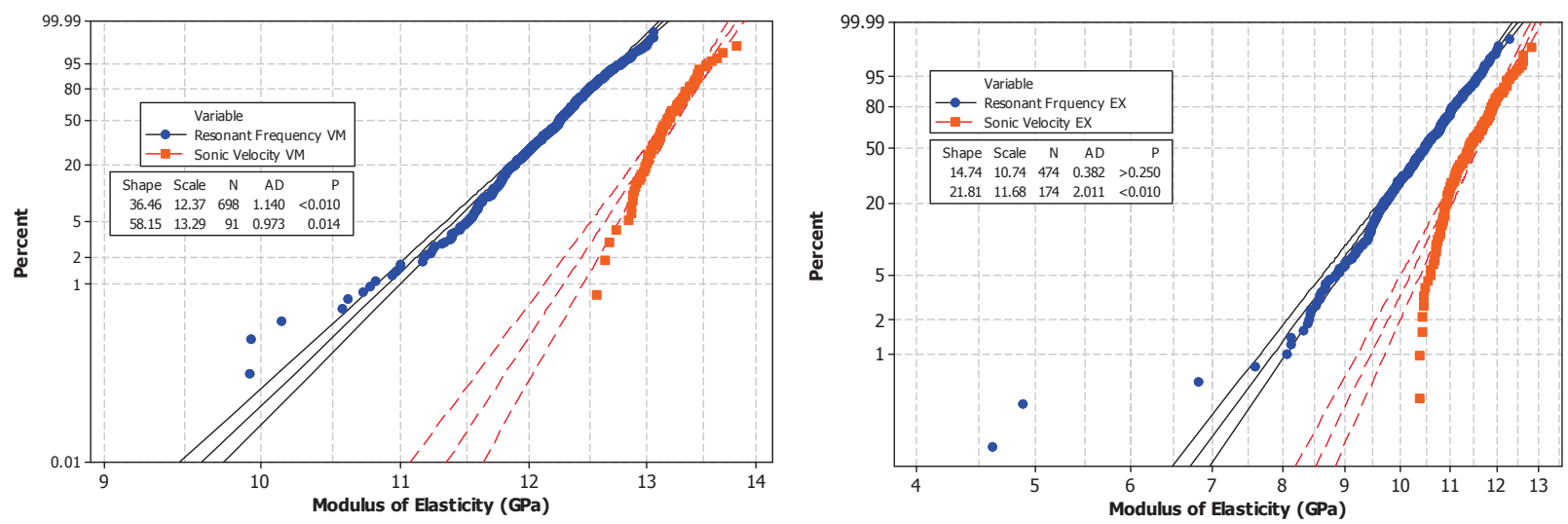

Figure 9. A comparison of the modulus of elasticity (Young's modulus) for vibration molded (left) and extruded (right) grades of graphite using two different measurement techniques. The modulus by sonic velocity exhibits considerably less variability and a lower incidence of low-value outliers.

\section{MECHANICAL PROPERTIES}

\subsection{Tensile Testing}

The qualitative tensile property comparison between billets of NBG-18 and PCEA is shown in Figure 10. A similar trend in variability from end to end can be seen in the extruded PCEA graphite that was seen in the density values. An overall comparison of tensile strength values via a probability distribution is shown in Figure 11, along with the relative differences between billets of the same grade. When considering the qualitative variability seen in Figure 10, further partitioning of the data into logical subsets will allow a more thorough determination of the degree of variability along this billet axis. Figure 12 (top) is a boxplot of the distribution of tensile strength values based upon groupings by slab, or layer along the long axis, which is one of the position axes that reveals a demonstrable within-billet gradient. 

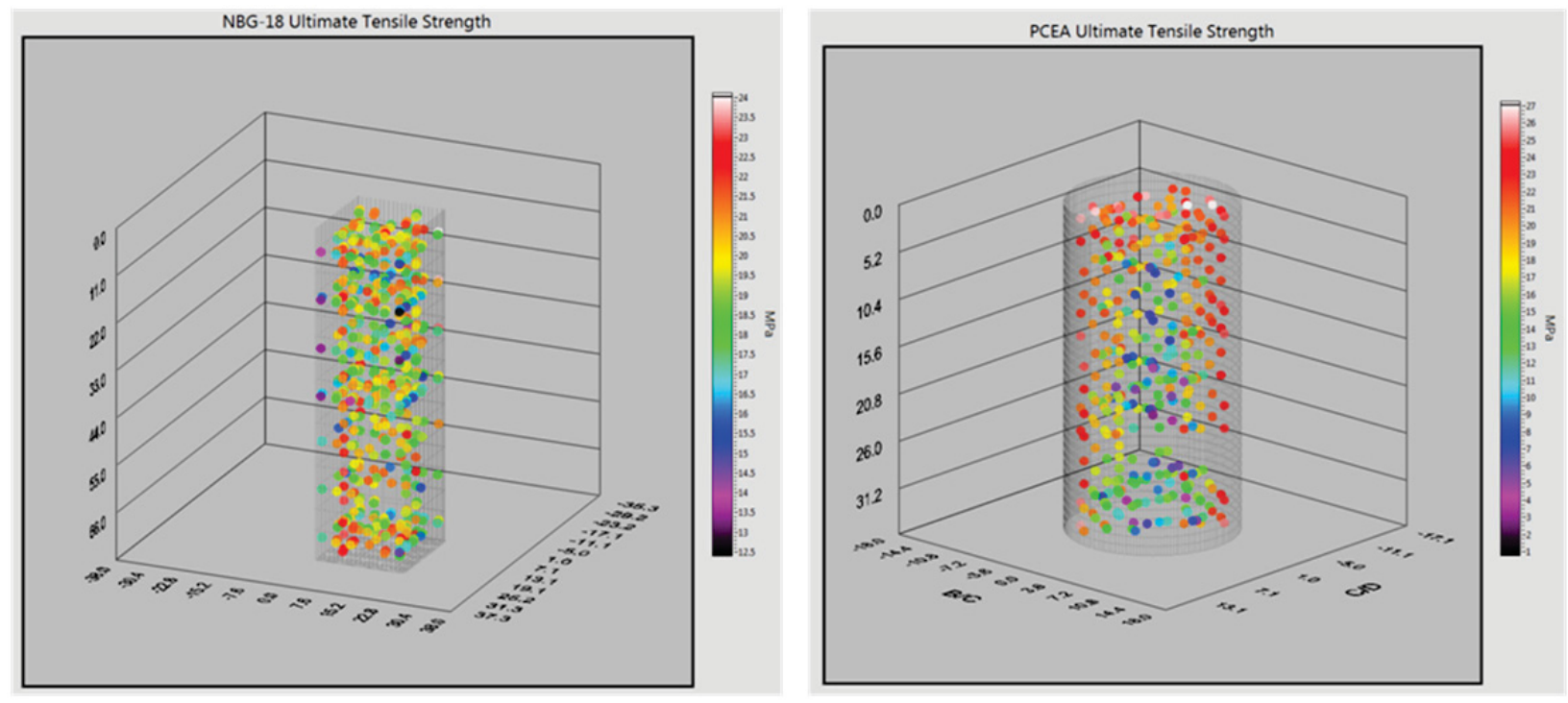

Figure 10. A qualitative 3D representation of the distribution of tensile strength values in vibration molded (left) and extruded (right) graphite grades. The gradient in the extruded graphite is reflective of the same pattern in the density distributions of Figure 4.

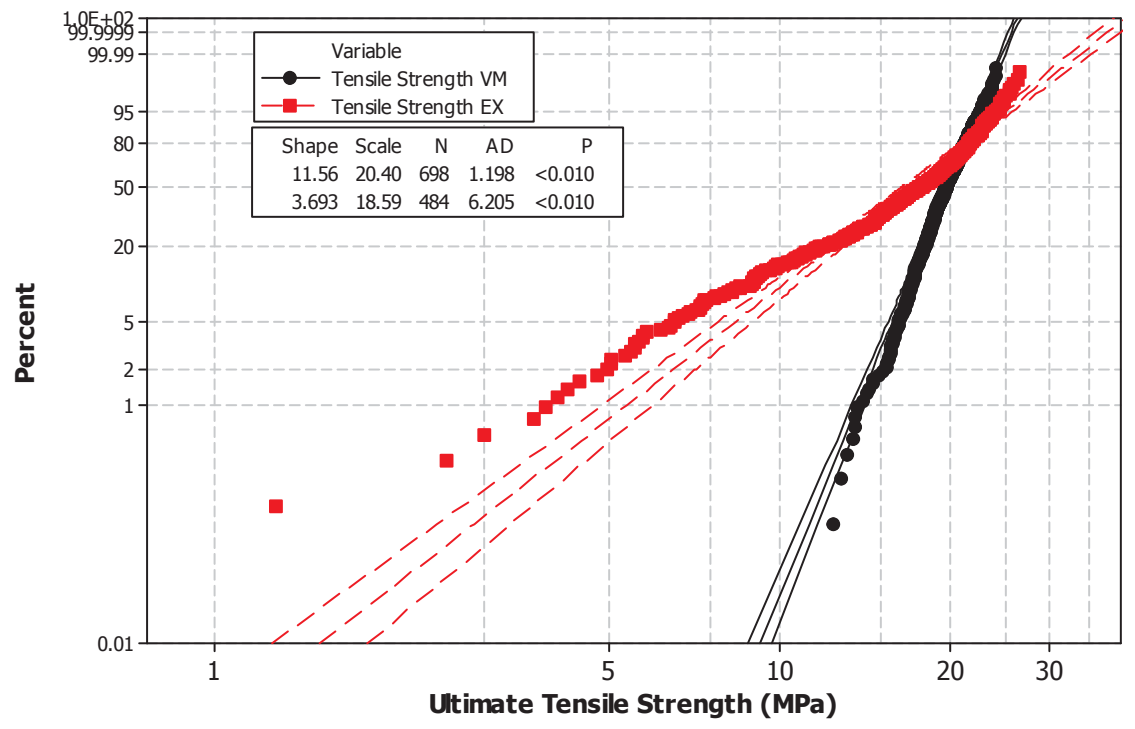

Figure 11. Weibull distributions of the tensile strengths measured in each grade show a higher characteristic value of 20.40 MPa for the vibration-molded grade vs. $18.59 \mathrm{MPa}$ for the extruded grade, despite the upper values for the extruded grade being higher. The overall distribution shows less variability at a higher overall mean value for the vibration-molded grade.

The same relative drop in tensile strength values from end to end can be seen based upon 7 distinct slabbased groupings obtained from the same positions in all three of the billets being evaluated. In order to ensure that the behavior is consistent across billets, the same evaluation can be made through further 

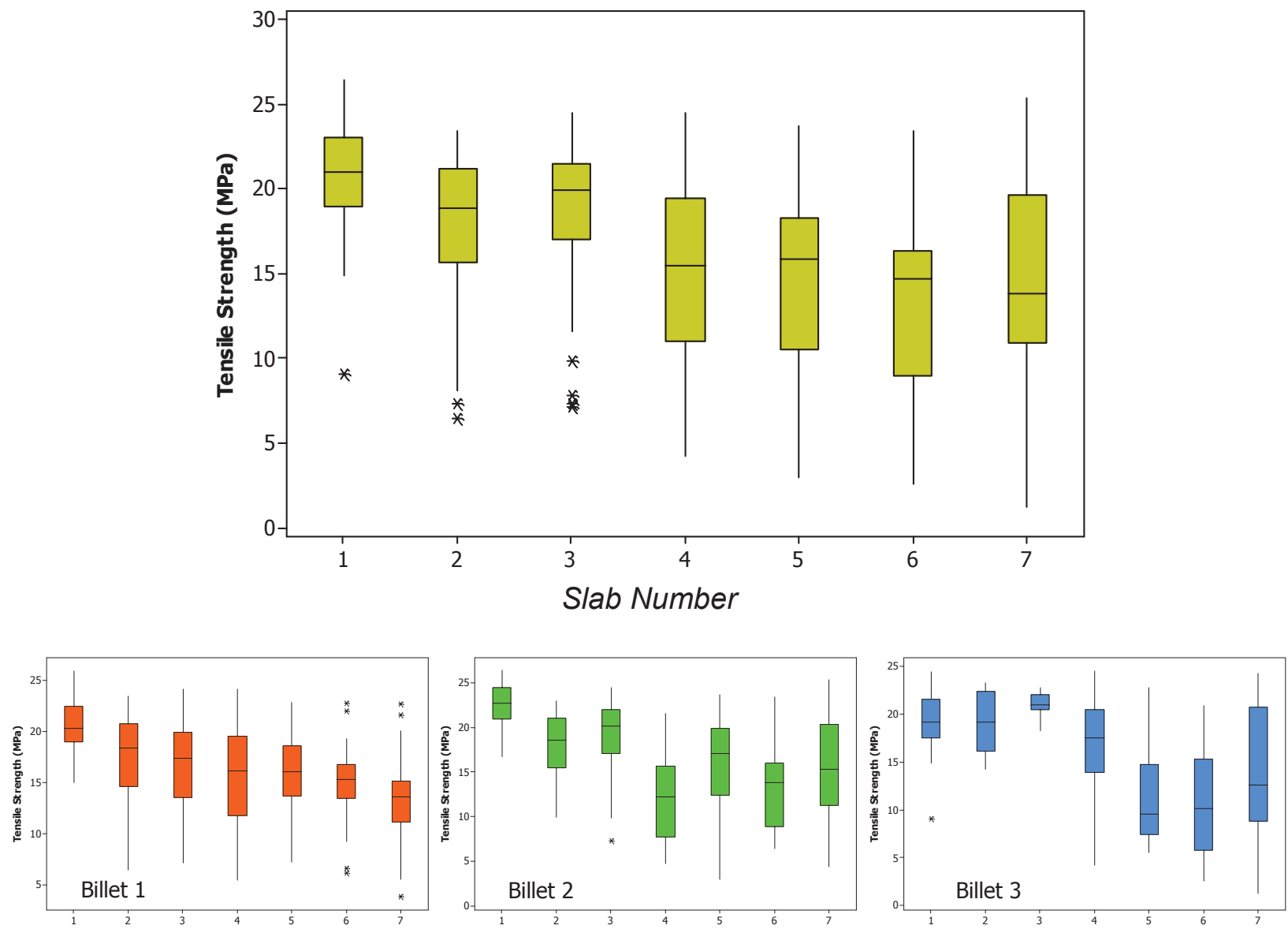

Figure 12. The distribution in tensile values from end to end in the extruded billet (represented by grouping the data as Slabs 1-7) indicates that the trend in decreasing value from "top" to "bottom" is consistent to varying degrees through each of the three individual billets (bottom 3 boxplots).

breaking down the subsets of data by individual billet, as is shown in the lower plots of Figure 12. While the general trend is confirmed, it can be seen that the extensive breakdown of data into smaller subsets begins to compromise the statistical significance of the resulting dataset. The trend in strength from end to end in the individual extruded billets is not as clearly discernible, as the variability in each dataset (represented by the height of the individual boxes/whiskers) increases along with the relative overlap between mean values in each of the slabs. In a complementary manner, the relative strength trend in the vibration-molded graphite that is not seen in similarly-sized data populations may be too subtle to be identified using the data collected to date, but, if it exists, may manifest itself as more data is collected on that grade and integrated into the full-grade representations. With the present tensile data collected on NBG-18 (698 tests), no statistically significant variation is seen.

The effect of orientation on mechanical properties is also important, as shown in Figure 13. The major orientations of interest from a global perspective in graphite are based upon the atomic level configuration of the graphite unit cell. The "with-grain" direction is along the $a$ direction, or plane of hexagonal carbon atoms, while the $c$ direction is perpendicular to the planes, in the direction of the stacked layers. In extruded graphite, the with-grain direction is assumed to be predominant along the 
extrusion axis. Vibration molding results in a settling of the planar orientations that is predominant along the short axis of the billet, which is orthogonal to the long axes as they rest on the vibrating plane during molding. For practical purposes, these orientations are captured in the datasets although the respective manufacturing processes result in nearly isotropic distributions. From a more application-specific evaluation, the comparisons are made based upon the major orientations within the original billets identified in Section 2.
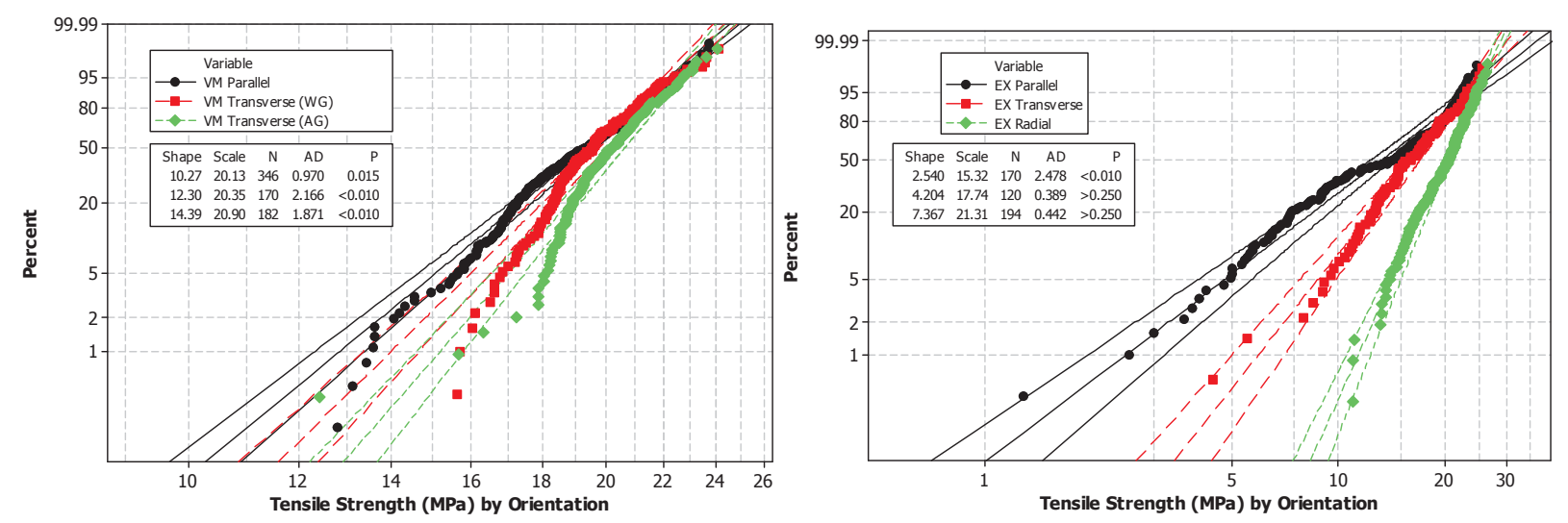

Figure 13. The variability between orientations is more pronounced in the extruded grade, with the radial orientation exhibiting the highest strength. The distribution of values is much closer in the vibration-molded grade, with the highest characteristic value for strength being shown by the orientation commonly regarded as the weakest - the against-grain orientation.

The Young's modulus of the material can also be evaluated through the direct stress-strain relationship captured during the execution of individual tensile tests, utilizing the two axes being plotted and applying the relationship $E=\sigma / \varepsilon$. Just as in Section 3, a comparison can be made between the test techniques used to capture the same material property - this time including the mechanical stress-strain response as a direct comparator to the physical property data captured through impulse excitation (vibration) or sound wave propagation. As is shown in Figure 14 (top), the stress/strain data yields the lowest stiffness values and highest overall variability, likely owing to the larger population of specimen flaws being captured in the gauge volume under test. 

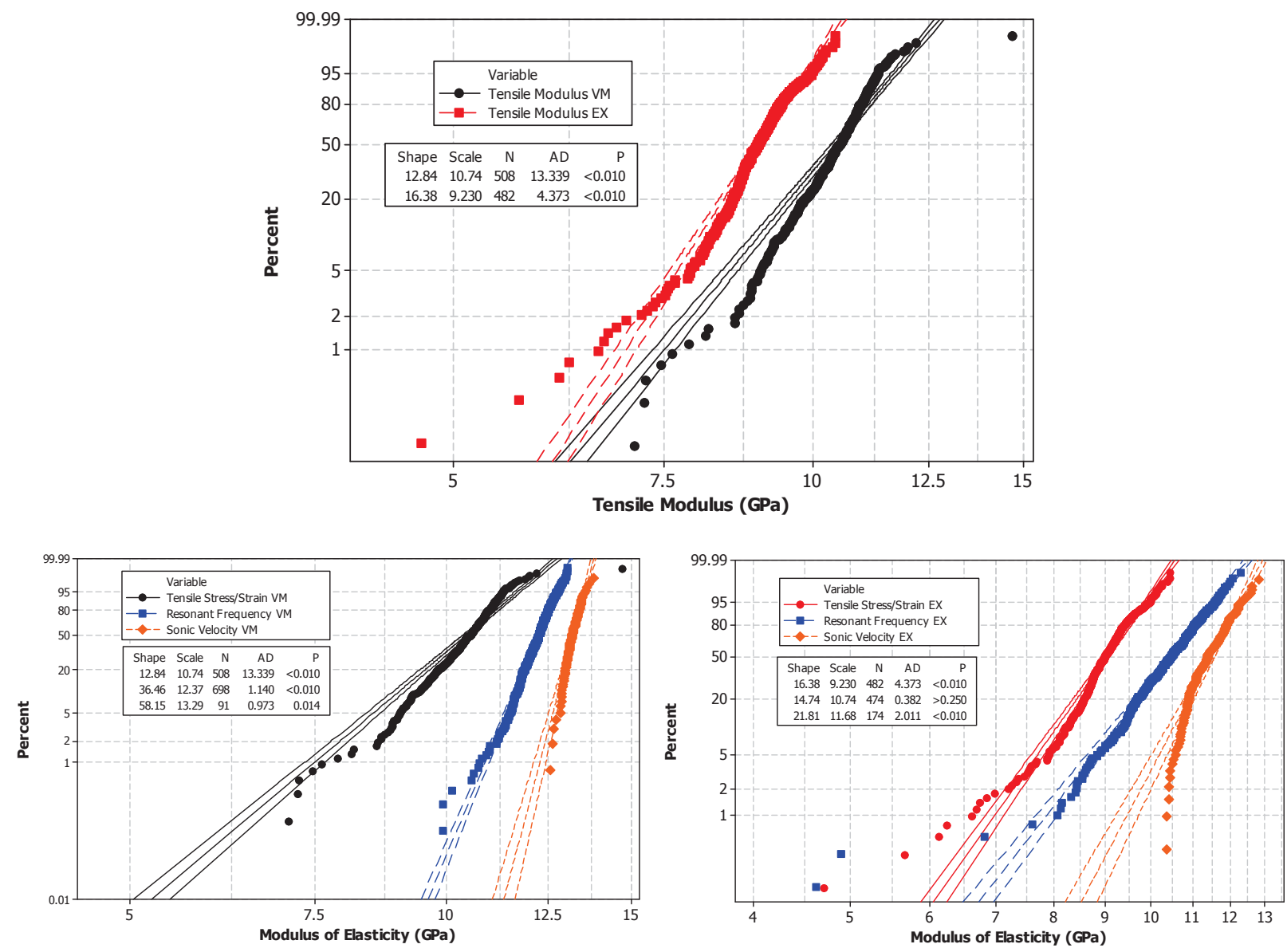

Figure 14. As with the physical property measurements, the vibration-molded grade exhibits higher stiffness across the distribution of values (top). The comparison of test techniques to resulting values further illustrates the effects of test method on the parameter being evaluated; the modulus by stress/strain measurements is consistently lower than the non-destructive techniques (resonant frequency and sonic velocity) presented in the previous section.

\subsection{Flexural Testing}

The qualitative comparison of flexural strength based upon the specific grades being evaluated and the relative positions within the billet is shown in Figure 15. As with the density and tensile strength results, the extruded grade shows a distinct property gradient through the volume of the overall billet. The associated probability distribution based upon the total amount of flexural strength data points for each grade is shown in Figure 16. The low-strength outliers are more prevalent in the extruded grade than in the vibration molded grade, and the variability in the dataset is much wider. There is a distinct crossover in flexural strength between the two grades, with a maximum flexural strength in the extruded grade at nearly $38 \mathrm{MPa}$ while the maximum flexural strength in the vibration-molded grade is just under $33 \mathrm{MPa}$. 

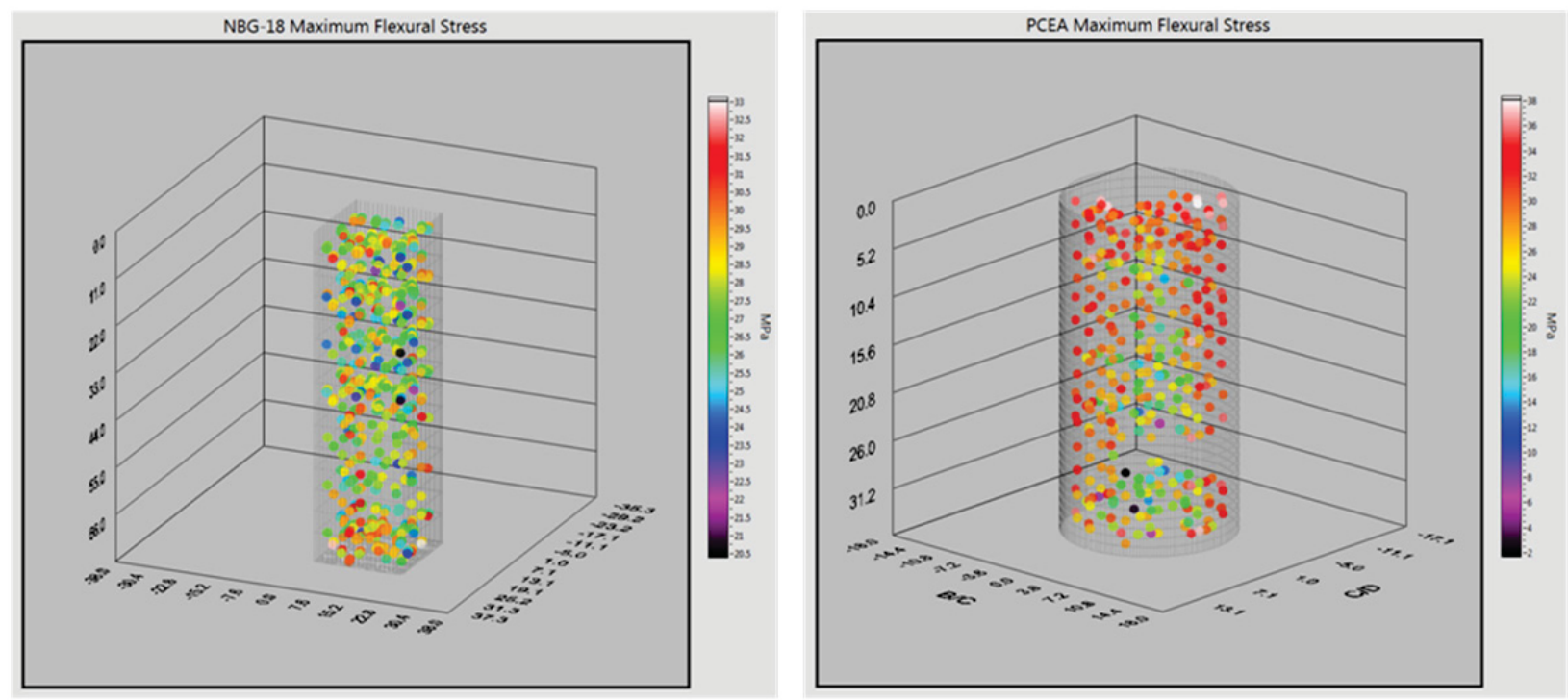

Figure 15. 3D representation of flexural strength results for each of the two grades shows a more homogeneous distribution for the vibration molded graphite than for the extruded grade, which further reflects the gradients seen in the density distribution of Figure 4.

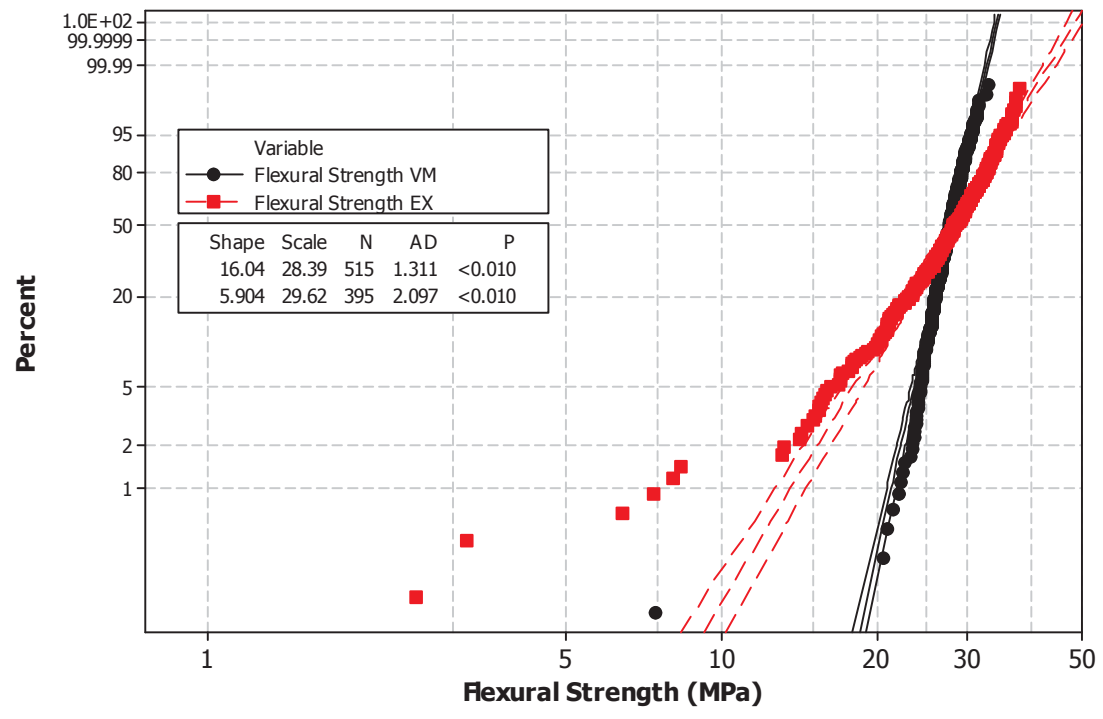

Figure 16. The flexural strength values are similar in distribution to the tensile test results, exhibiting a crossover region at the higher values for the extruded graphite. Unlike the tensile results, the characteristic value for flexure strength is higher for the extruded grade despite the larger range of variability in that grade, particularly at the low strength values. 

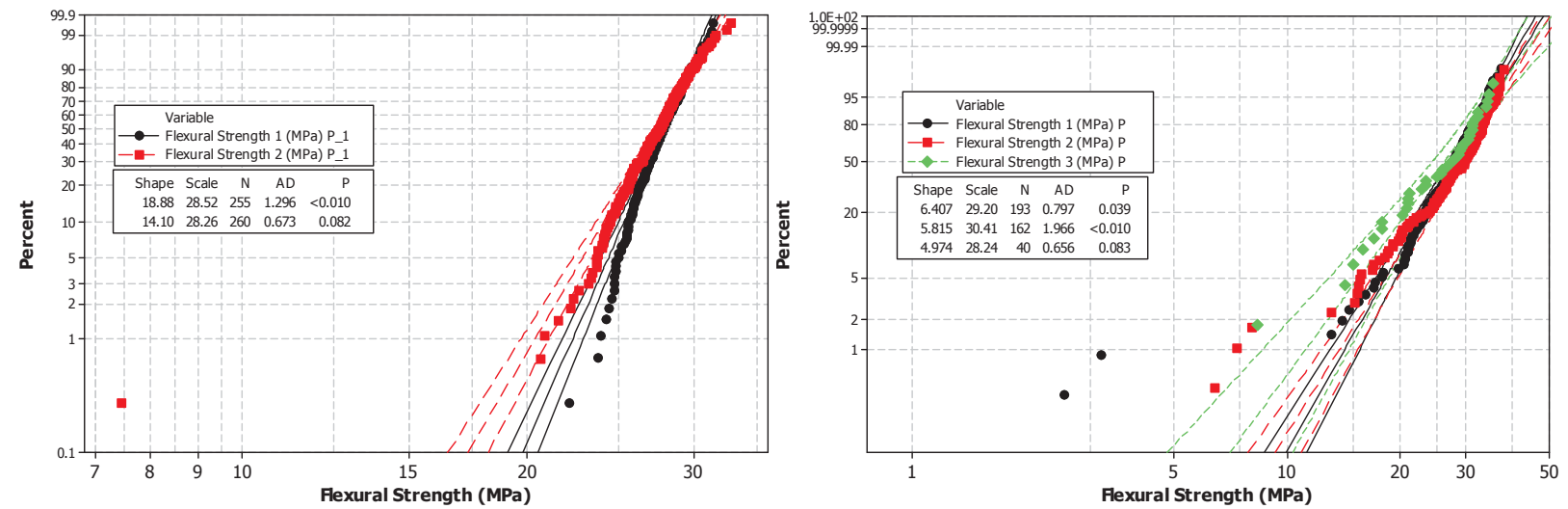

Figure 17. Flexural strength between billets of the same grade for two vibration-molded (left) and three extruded (right) graphite billets.

The within-grade variability is shown in Figure 17. As can be seen from the figures ${ }^{\mathrm{a}}$, the variability (slope) of the distribution is consistent between grades, and reflective of the overall distribution seen in the total distribution plot shown in Figure 16. Outside of the single low outlier value exhibited in the second NBG-18 billet, the probability distributions are relatively consistent within the vibration-molded grade when compared to the extruded grade.

The shift in flexural strength values from billet end to billet end (slab number) is also seen in the extruded graphite to an extent not seen in the vibration-molded grade. Figure 18 shows comparison boxplots of flexural strength as grouped by $z$-axis slab number that reflect the decrease in values from one end to the other. Although the shift is not as distinct from end to end as seen in density and tensile strength grouped values, it is clearly prevalent when compared to the same boxplot for the vibrationmolded grade, which shows no significant variability in mean values. An analysis of variance (ANOVA) evaluation (Figure 18, lower right) shows an even more clear decreasing trend in values through the length of the billet. The associated P-value for mean values of flexural strength by slab in the extruded billet is well below 0.05 (95\% confidence interval), indicating that a difference in the mean values based upon this decreasing trend is statistically significant.

A comparison of orientation effects between the two grades is shown in Figure 19. The relative variability between orientations in the vibration-molded grade is fairly consistent; both the characteristic values and distribution slope of each is similar. More disparity exists in the extruded grade, with the radial orientation having the most consistent and highest mean flexural strength values. As with the parallel orientation in tensile testing, the parallel orientation exhibits the highest amount of variation and the lowest mean strength in flexure.

\footnotetext{
${ }^{\text {a }}$ Note that the strength data from a third billet of NBG-18 has been collected, but only the physical property and tensile test data results are included in this report. Flexural and compressive data from this third billet will be included in the continuing overall evaluation pending the validation and release of those particular datasets.
} 

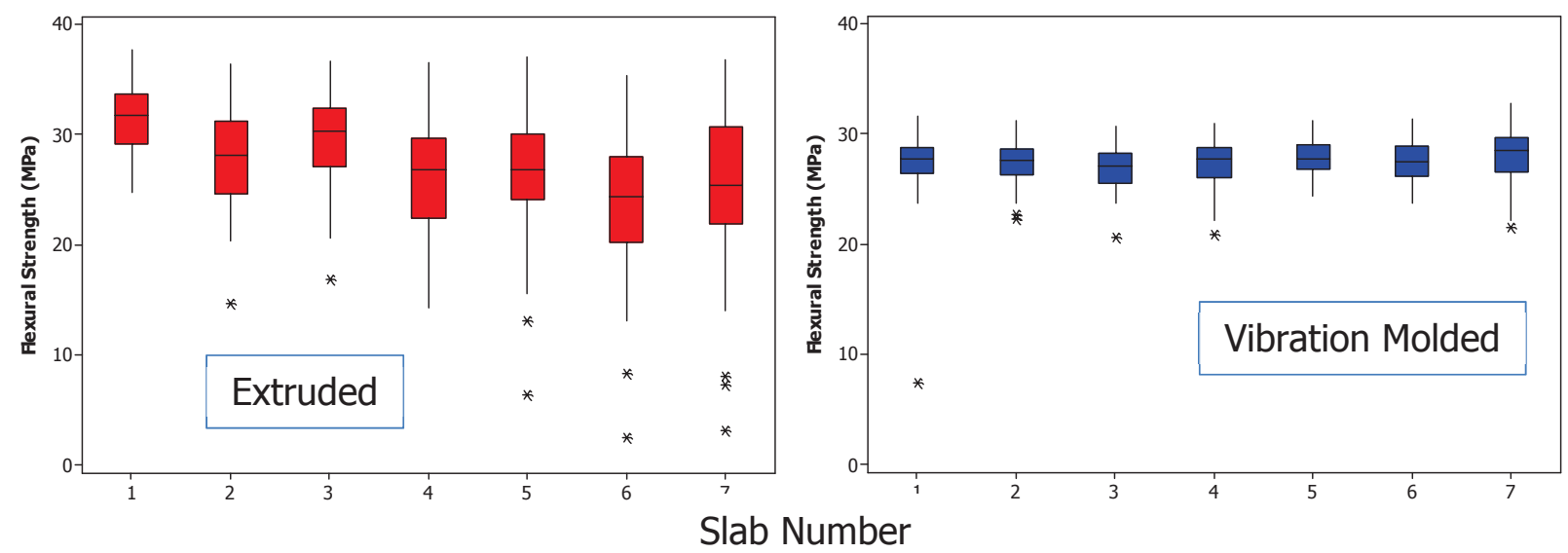

Figure 18. The end-to-end variability and general spread in data is clear in the extruded grade (top left), with values decreasing by zaxis length via slab number groupings. The vibration-molded grade (top right) exhibits a similar mean value throughout. The decreasing trend in mean values is confirmed by the stacked ANOVA (right).
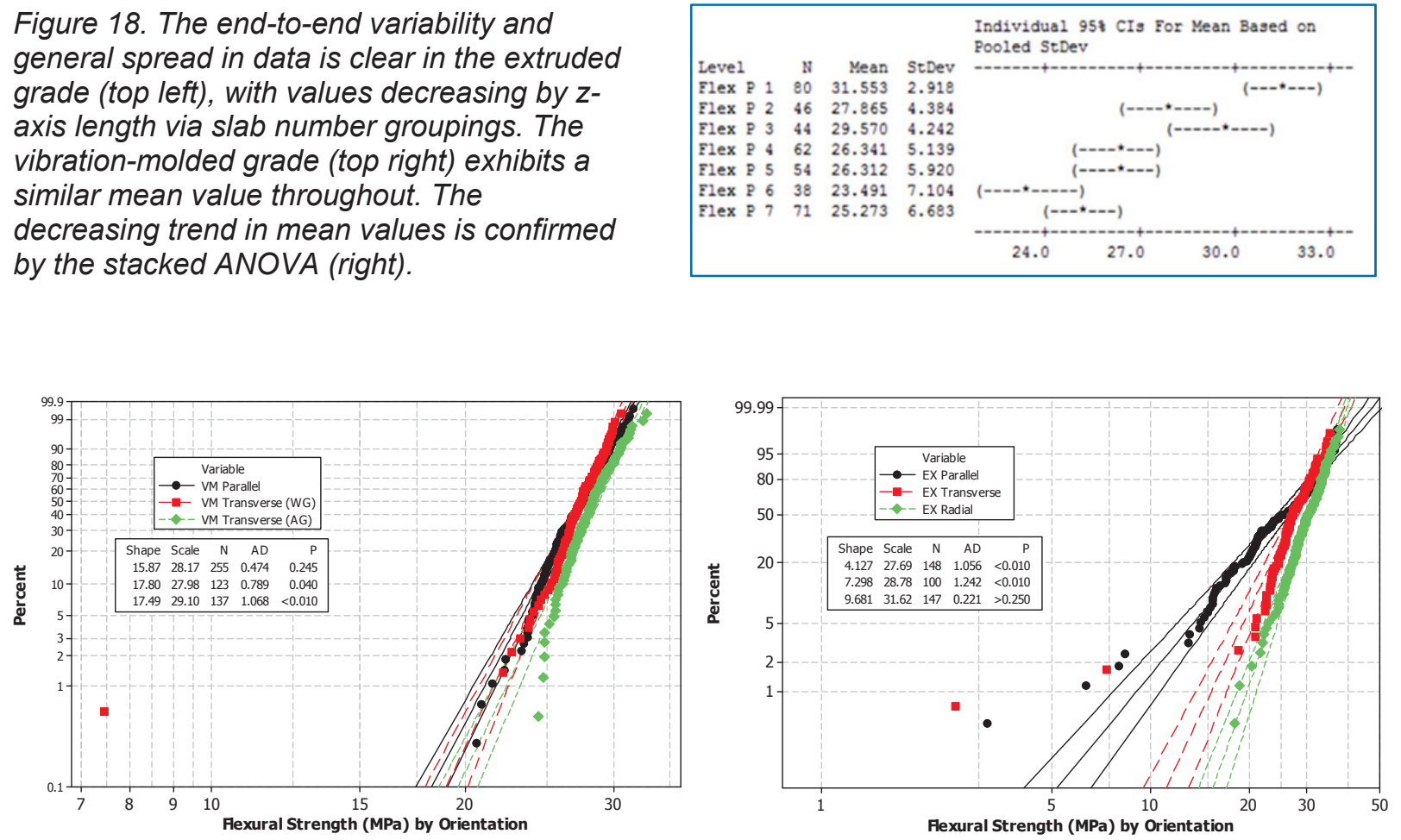

Figure 19. Flexural strength by orientation in each of the grades exhibits similar results to the tensile data - in the vibration molded graphite, the results in each of the orientations is similar, with the against-grain orientation showing the highest strength value for the distribution. The radial orientation is the strongest in the extruded grades (right), while the parallel orientation is weakest. 


\subsection{Compressive Testing}

A similar qualitative comparison of compressive strength to that shown for both tensile and flexural strengths, demonstrating the comprehensive value distributions based upon the specific grades being evaluated and the relative positions within the billet, is shown in Figure 20. As with the other mechanical
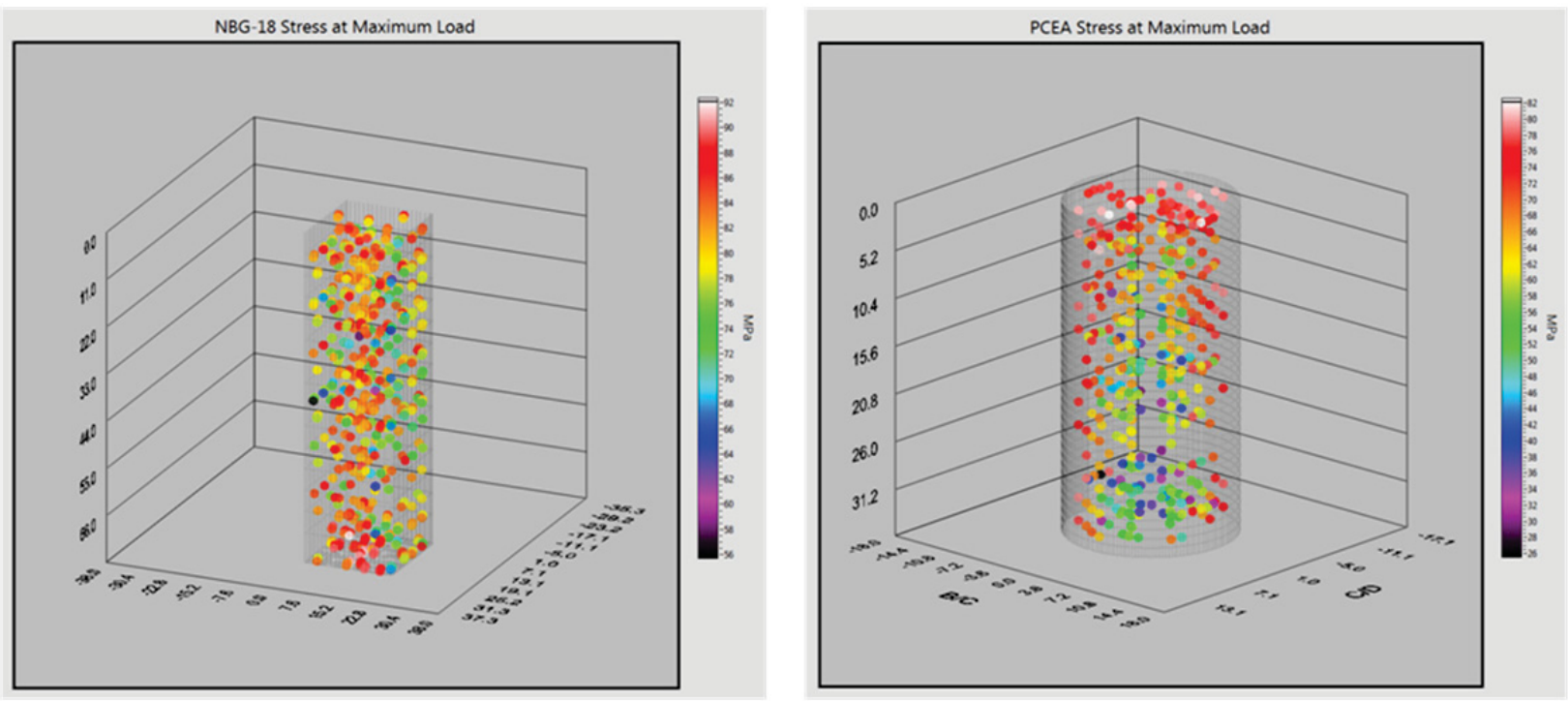

Figure 20. The 3D representation of the compressive strength values for each of the two grades shows a similar qualitative distribution to that seen in the previously evaluated properties, with the compressive strength exhibiting an end-to-end and center-outside gradient.

test values, the pattern that emerges is a relatively homogeneous distribution of values in the vibrationmolded grade, with an end-to-end and inside-outside decreasing strength trend in the extruded grade, particularly along the centerline of the billet. The overall distribution of compressive strength values is shown in Figure 21. A number of key features are captured in this figure; first, as in other graphites, the compressive strength is significantly higher than that in tension or bending, with mean values for stress levels increasing by a factor of roughly 2 to 4 of the tension-based failure of tensile and flexural testing. Second, similar to the tensile and flexure test results, the data scatter is significantly higher in compression for the extruded grade, with a variability shape factor (slope) that decreases from just under 20 for the vibration-molded grade to 7 for the extruded grade. Unlike the tensile and flexure results, no crossover in maximum values exists between the two grades - the vibration molded graphite is higher in compressive strength throughout the data distribution. Both the maximum and minimum values are higher for the vibration-molded grade. 


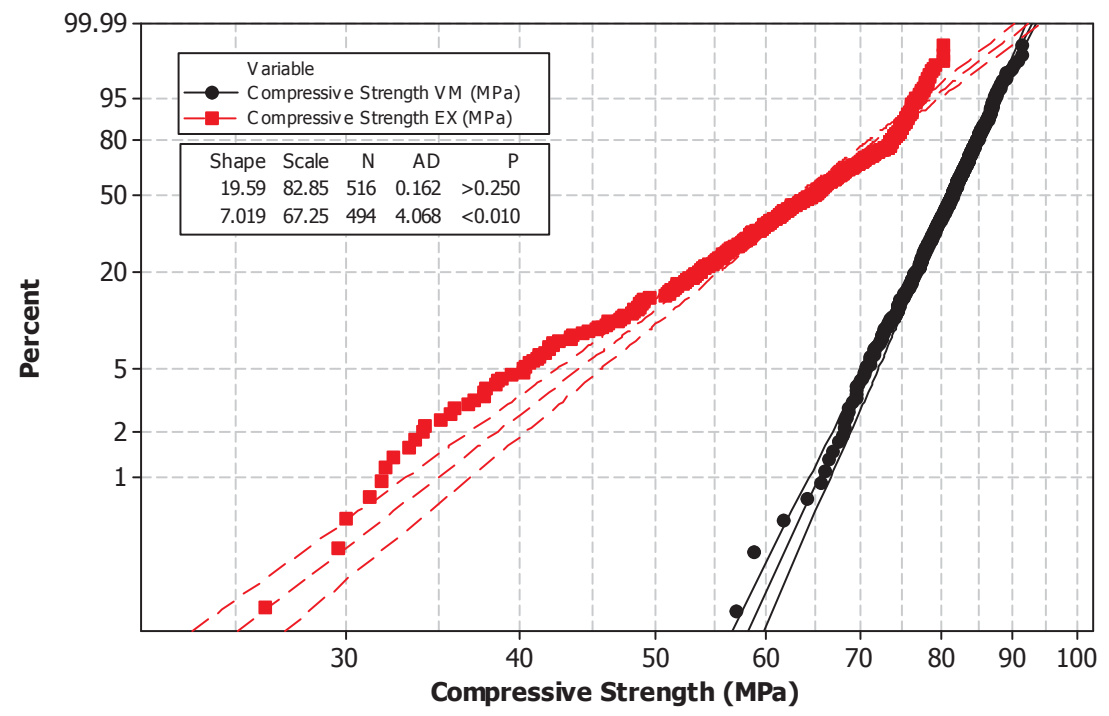

Figure 21. Compressive strength values for the billets tested show greater variability and a lower overall strength distribution in the extruded grade.

The same-grade billet-to-billet variation is shown in Figure 22. As shown in the figure legend, the shape factor actually shows a larger disparity in specific values between the two billets of vibrationmolded graphite than is seen for the three billets of extruded graphite. Despite this, the variability is considerably less overall and within the individual billets for the vibration-molded grade as evidenced by the considerably smaller spread in the distributions, ranging from just under $60 \mathrm{MPa}$ to just over $90 \mathrm{MPa}$ in the vibration-molded graphite and from approximately $30 \mathrm{MPa}$ to $80 \mathrm{MPa}$ for the extruded grade. A larger spread in compressive strength values is also evident at the lower end of the strength range for the extruded grade than for the vibration-molded grade; the vibration-molded graphite remains reasonably linear throughout the range of values even when the low outlier strength levels are included.
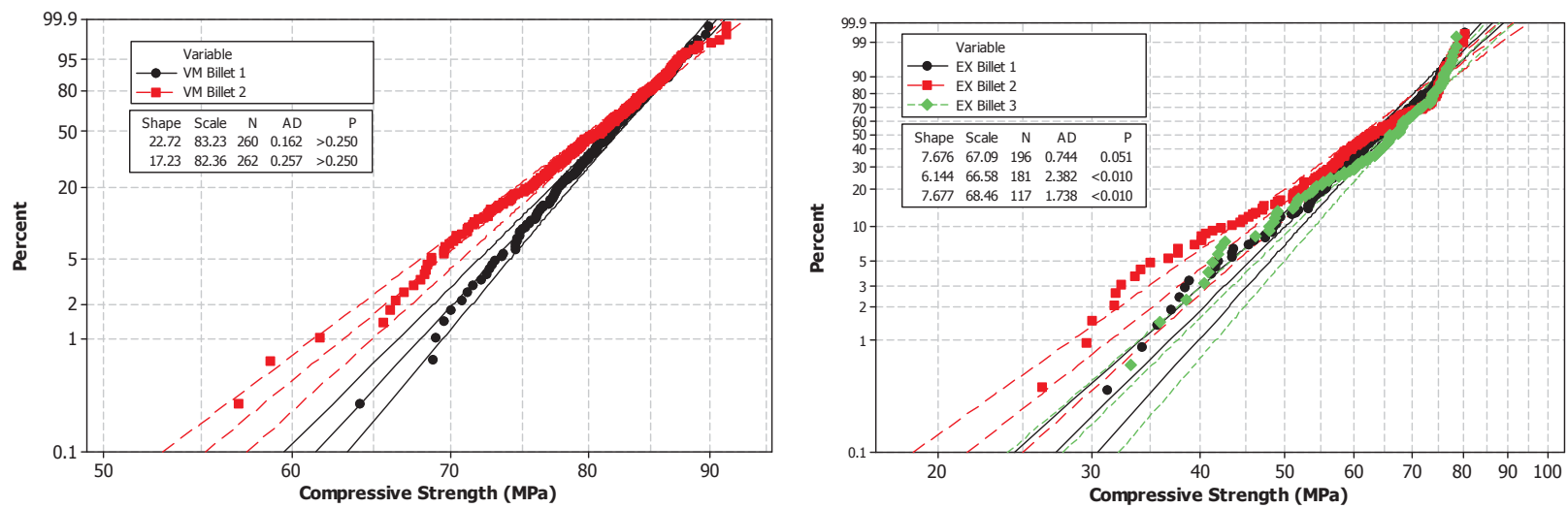

Figure 22. Compressive strength distributions for the vibration-molded billets (left) and extruded billets (right) indicate a narrower range of variability for individual vibration-molded graphite than for extruded. 
The orientation effects reveal a slightly different response in the vibration-molded grade when tested in compression compared to the results in tension or flexure. As is shown in Figure 23 (left), the againstgrain orientation shows the lowest mean strength level despite being very close in value to the other
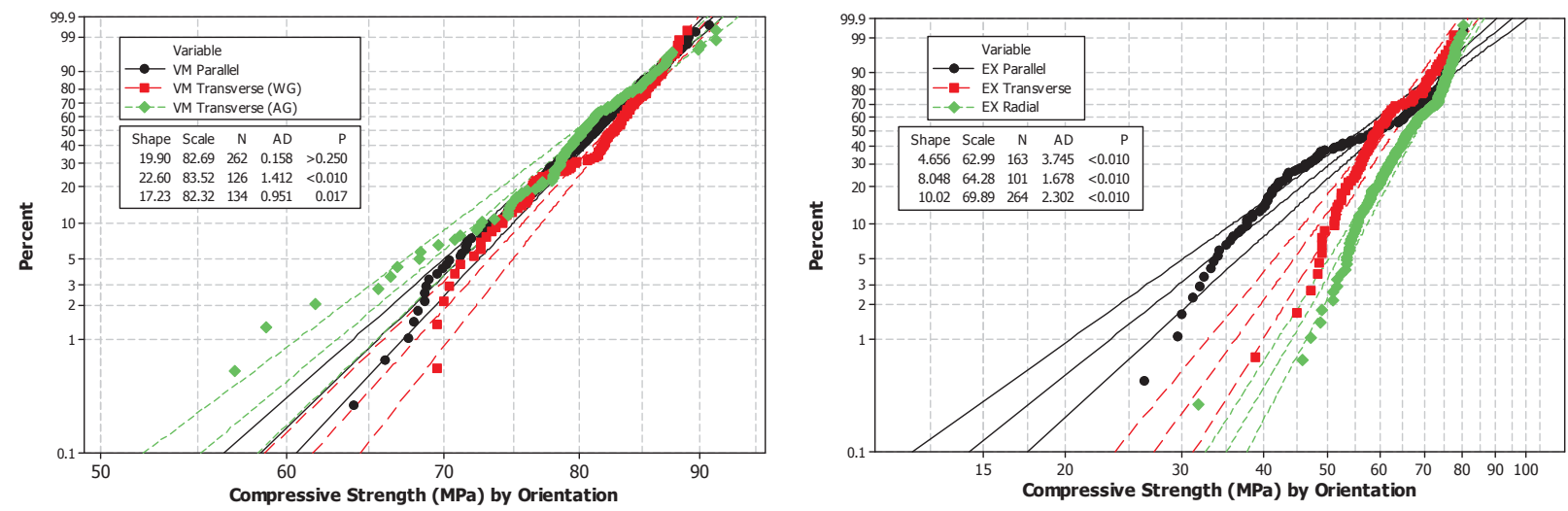

Figure 23. The compressive strength distributions by orientation overlap are consistent at the upper values for the vibration-molded graphite (left), with higher variability and lower overall values for the against-grain orientation in the lower range. The extruded grade (right) shows the same pattern for mechanical strength as seen previously - the radial orientation is the most consistent and strongest orientation, while the parallel orientation has the weakest distribution of values.

orientations. The lower range of the distribution also exhibits more scatter than was seen in the tensile and flexural test results, seen qualitatively in the sharp decrease in slope for the against-grain orientation for the data values below the $10^{\text {th }}$ percentile of the distribution. By contrast, the extruded grade exhibits behavior very similar to that seen in the other mechanical test results, with relatively non-linear Weibull distributions and considerably less strength in the parallel orientation than is seen in the other two orientations. As with the tensile and flexural strength tests for the extruded grade, the radial orientation exhibits the highest overall strength distribution.

The main within-billet variability by $z$-axis or Slab position for the extruded grade identified in the density distributions and confirmed in the other mechanical test results is shown in Figure 24. As can be seen from the boxplot, the trend in variability follows the same pattern from end to end as seen in the other results. The lowest mean values are at the end of the billet opposite the highest mean values with a gradual transition through the entire $z$-axis range. The uppermost range of values is clearly in the layer designated as Slab 1. 


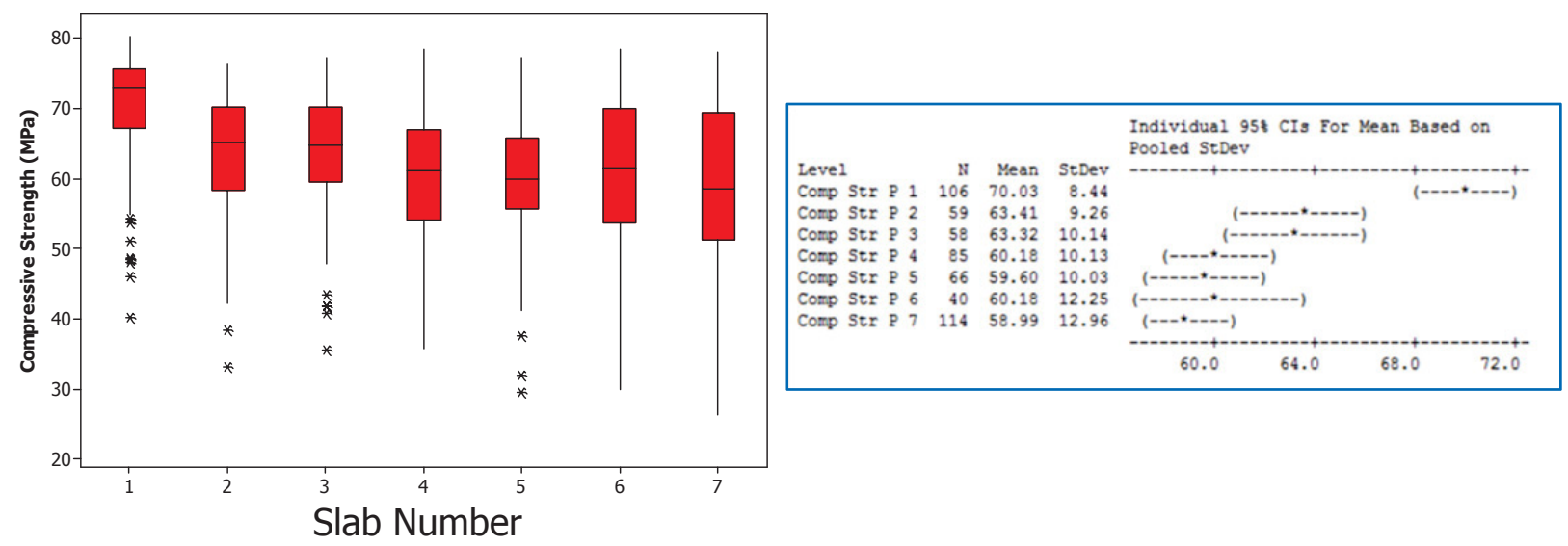

Figure 24. The end-to-end trend variation in the extruded grade shows the same trend in decreasing values by slab number (left) with confirmation of the variability in mean values shown by an ANOVA evaluation (right).

\section{DISCUSSION}

A full breakdown of the property variation is each grade can clearly be broken down into subgroups that yield differences with far greater resolution than is presented by the relatively simple $z$-axis groups evaluated in the previous sections. The density and property variation in the extruded graphite, for instance, shows gradients in the transverse directions from the centerline as well as the $z$-axis gradient from end to end that was plotted in the previous sections for each of the measured properties. As was seen in Figure 23, the radial orientations had a higher overall strength distribution, so this qualitative observation may be based largely on the artifact that the stronger radial orientation specimens can be extracted closer to the outer edge of the billet. The distribution could therefore be effectively skewed toward larger strength values at the outside positions even though the relative strength of each orientation with respect to distance from the billet centerline might actually remain consistent. Using the compression testing results as an example and the radial orientations as a specific response group, this variation and/or presence of a property artifact is readily evaluated by plotting the mean values for compressive strength for the radial specimens by position rows, which are numbered 1 through 5 as the distance from the billet centerline increases along the $x-y$ plane to the outer billet edge. The boxplot in Figure 25 confirms the higher values at the outermost positions (Row 5) throughout the range of $z$-axis groups even with the orientation effect isolated, confirming that the property gradient along the transverse billet axis exists and is not an artifact of the physical limitations of removing specific geometries at fixed orientations close to the outer edge of the billet. Further breakdowns within these subgroups can be made based upon a qualitative evaluation of the 3D property plot for compressive strength. The grouping within the billet (Figure 26) that shows the highest distribution of strength values appears to be represented by the entire population of Slab 1 (billet top), while the lowest values for compressive strength are grouped in the "bottom" slabs near the centerline. 


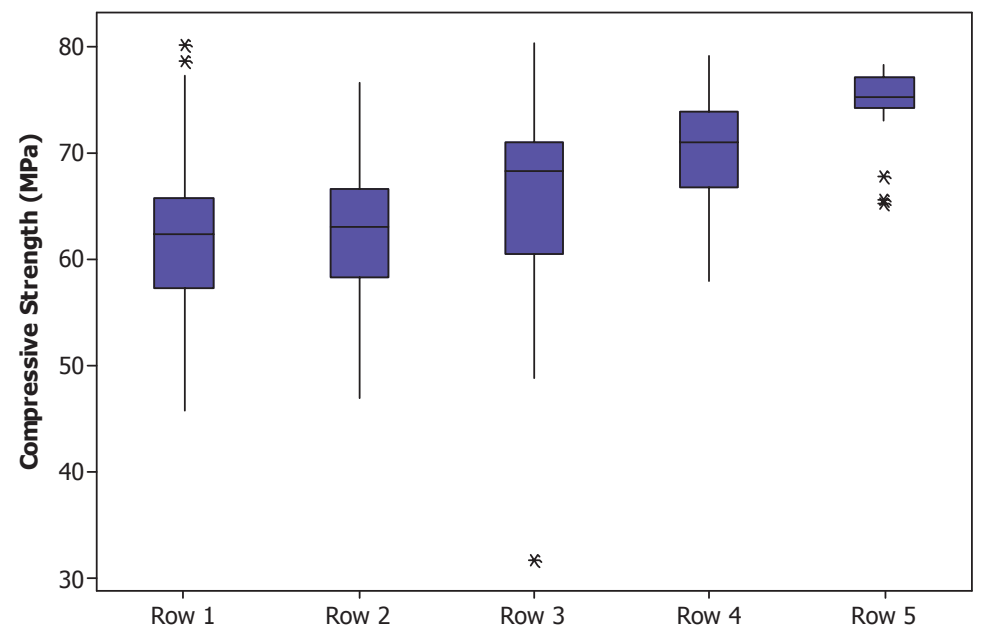

Figure 25. Boxplots confirm the increasing trend in strength values in the radial orientation from centerline to the outer edge of the extruded grade seen qualitatively in the 3D plot of Figure 20.
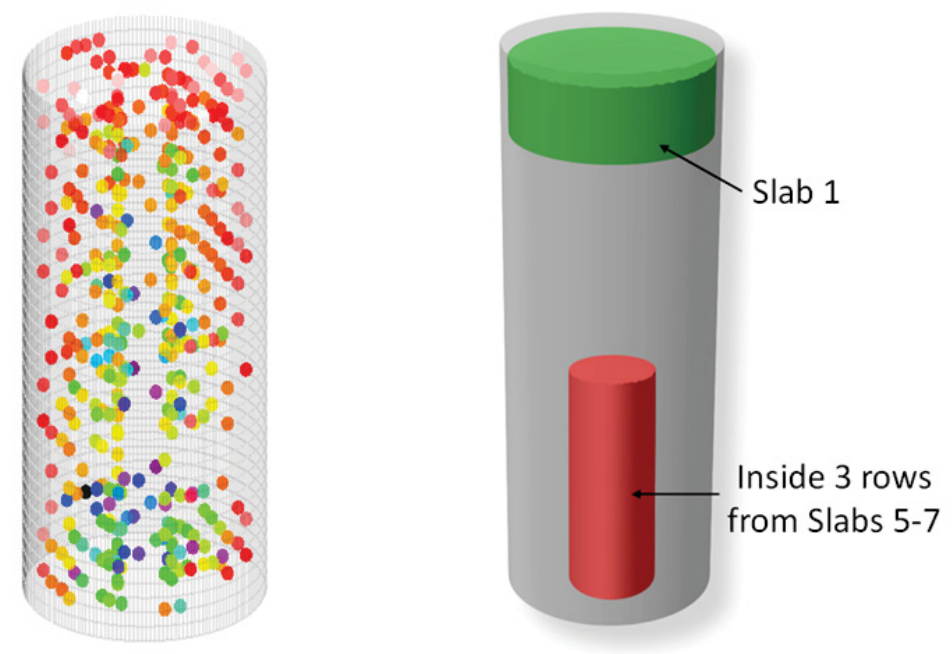

Figure 26. The 3D plot (left) high strength distribution in the upper layer of the extruded billet and a lower strength region in the bottom center section of the billet. These regions can be quantified by extracting data from the subgroups that compose those specific positions in each of the billets (right).

These two distinct groupings, represented by the green and red sub-sections in Figure 26 (right), demonstrate the global variability in properties within billets of the extruded grade from a more practical application and component-level understanding than would direct comparisons of high and low scalar values with error percentages. Figure 27 shows a boxplot (a) of the two "extremes" of the grade 

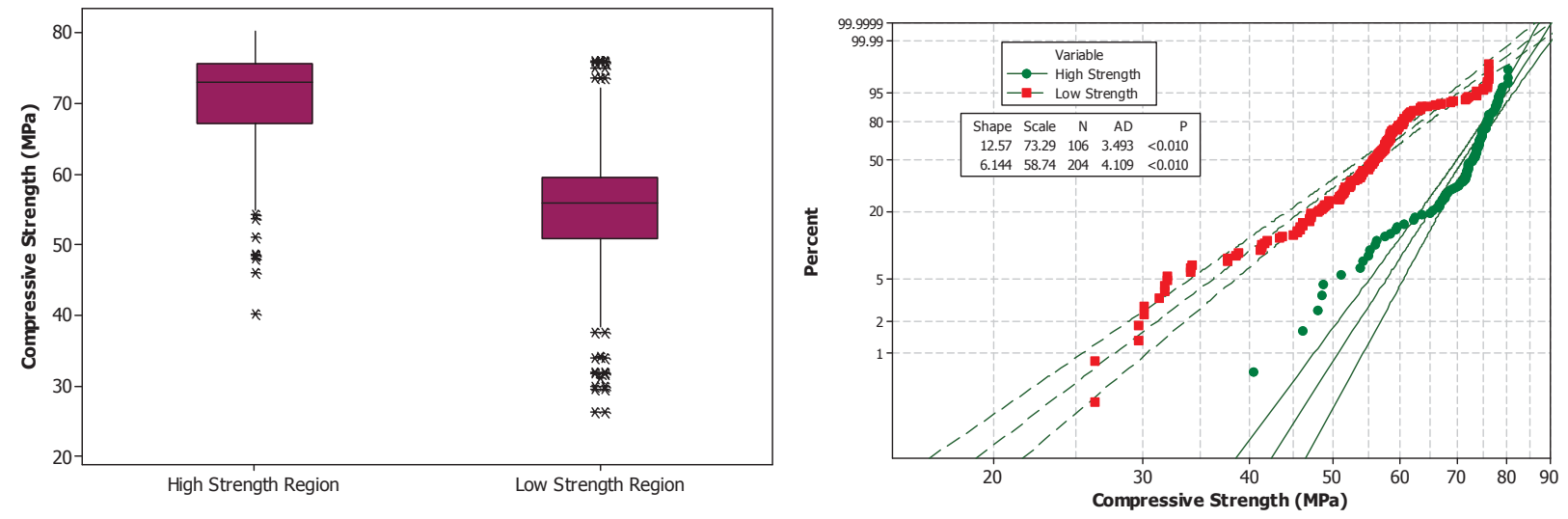

Figure 27. A boxplot of the two regions (left) shows the difference by directed subgroup of the variation from high strength to low strength regions in the extruded graphite. The specific distribution of strength values from each of those regions (right) provides confirmation of the variation and characteristic value levels.

distribution along with a probability distribution (b) comparing the two groups. Further confirmation of the property relationship to the density distribution is enabled by the large disparity between the two sets of data from within individual billets. The observation that density is a key predictor of properties is not surprising; the variation in measured values is a reflection of the pore, void, and crack population inherent to graphite volumes. The size, shape, and orientation of the larger "disparate flaws" will also have a distinct effect on measured mechanical properties, even when those flaws result in no actual test data being collected. The within-billet variability of the largest disparate flaw population can be represented through careful tracking of specimens that do not reach the property evaluation phase at all, as finished test specimens from prescribed positions are not possible due to flaws that compromise the specimen coupons prior to completion of the machining process. Large flaws that are on a scale that traverses a considerable fraction (or, in some cases, the entirety) of a coupon or partially machined specimen will result in sample breakage under handling stress. Because these specific positions are tracked using the same ID codes that track test specimens, a representation of the large disparate flaws can be plotted in the same manner as other properties of interest. Figure 28 shows the individual positions that were not machined into test specimens due to failure during the handling/extraction/machining process. As can be seen from the representation, the largest disparate flaws are grouped in the same region as the finished specimens with the lowest density and lowest strength values. It is not necessarily the large individual flaws themselves that compromised whole specimens and weakened neighboring specimens so much as it is an indication of a wider distribution of flaw sizes in those regions that are large enough to compromise measured strength or physical property values. 


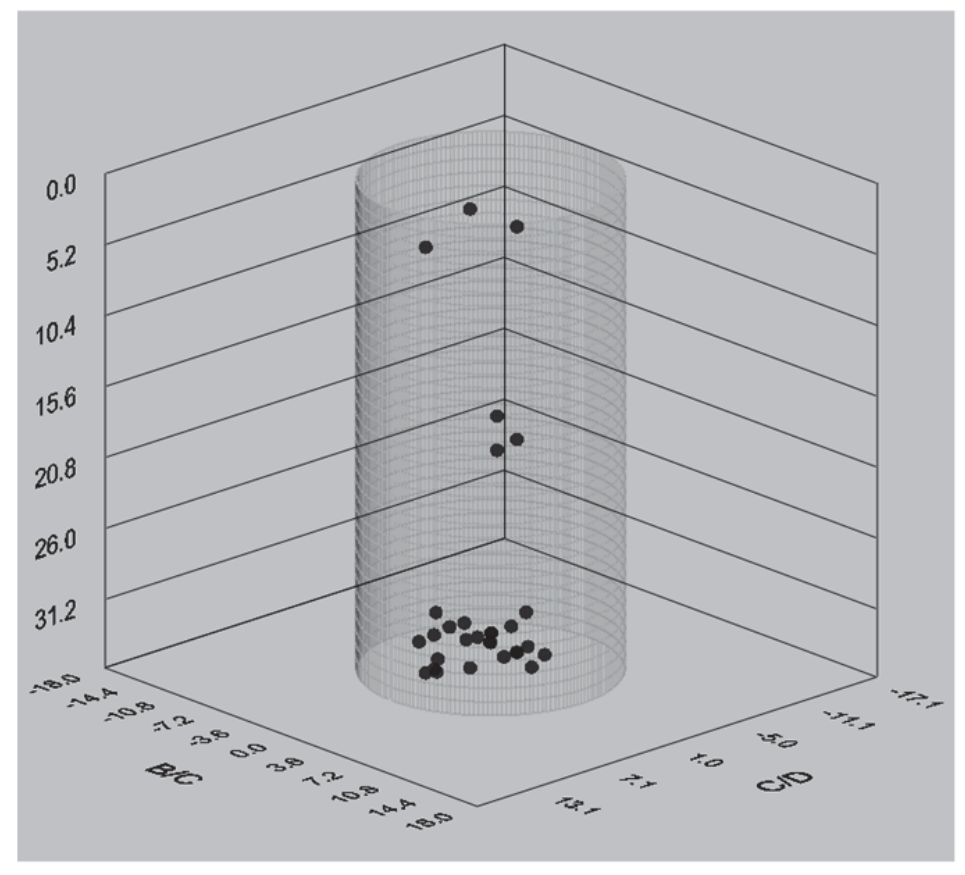

Figure 28. Distribution of the largest disparate flaws based upon the resolution of individual extraction and handling of specimen coupons.

The potential for the continued development of flaw distribution/property relationships becomes more distinct as data continues to be collected and evaluated utilizing similar subgrouping techniques based upon qualitative observations. An understanding of the dependency of strength and performance mechanisms on grade-specific characteristics (raw materials and process techniques) is critical to the optimization of graphite in nuclear-based systems, and this understanding begins with the identification of property value variations that are demonstrated to be statistically significant.

\section{SUMMARY}

The comparative strength and elastic properties of grades NBG-18 and PCEA have been compiled based upon the data collected thus far in the Baseline Graphite Characterization program. From the 3,142 mechanical test specimens that have been tested in various forms to date, a number of graphite response characteristics have been quantified that help to elucidate the overall graphite behavior in the asmanufactured state.

The mean strength and modulus values are higher in the vibration-molded graphite (NBG-18) than the extruded grade (PCEA) in all of the properties evaluated in this report except flexural strength, and are considerably more predictable based upon the relative data scatter between the two grades. The distribution of property values based upon position within the original as-manufactured billet is also much more consistent throughout the vibration molded billet than the extruded billet. A qualitative analysis of properties through 3D representations is confirmed through quantitative analyses based upon Weibull probability distributions and comparisons of mean values based upon grade-and billet-specific subsets of property values. The basic manufacturing variables in nuclear-grade graphite, filler type, particle size, and 
compaction process, are compared side by side and indicate distinct regions of property gradients within the extruded graphite that are not as clear from the relatively homogenous property distributions within the vibration-molded grade. The likely effect of those manufacturing variables on flaw size, density, and distribution in the final billet is apparent from a comprehensive evaluation of property values.

The resolution of distinct subsets of data, as have been analyzed in this report, will continue to evolve as the number of data points representing physical or mechanical property values continues to increase. Data is still being collected on these grades as well as other candidate grades of graphite that differ in raw material, particle size, and manufacturing process. Further evaluation in this program will rely on larger and larger datasets that allow conclusions to be drawn concerning the design basis for utilization of specific grades in nuclear applications.

\section{REFERENCES}

1. Hindley, P., et al "A numerical stress based approach for predicting failure in NBG-18 nuclear graphite components with verification problems," Journal of Nuclear Materials 436, pp. 175-184 (2013).

2. Becker, T., Marrow, T., Tait, R. "Damage, crack growth and fracture characteristics of nuclear grade graphite using the Double Torsion technique" Journal of Nuclear Materials 414, pp. 32-43 (2011).

3. Petti, D. "R\&D Plans and Accomplishments," presented at the VHTR R\&D FY10 Technical Review Meeting, Denver, CO, April 27-29 (2010).

4. "NGNP Project: 2011 Status and Path Forward," INL/EXT-11-23907, Idaho National Laboratory, December 2011.

5. ASTM Standard C749-08 "Standard Test Method for Tensile Stress-Strain of Carbon and Graphite" ASTM International, West Conshohocken, PA (2010).

6. ASTM Standard C651-11 "Standard Test Method for Flexural Strength of Manufactured Carbon and Graphie Articles Using Four-Point Loading at Room Temperature" ASTM International, West Conshohocken, PA (2011).

7. ASTM Standard C695-91 "Standard Test Method for Compressive Strength of Carbon and Graphite" ASTM International, West Conshohocken, PA (2010).

8. ASTM Standard C747-93 "Standard Test Method for Moduli of Elasticity and Fundamental Frequencies of Carbon and Graphite Materials by Sonic Resonance" ASTM International, West Conshohocken, PA (2010).

9. ASTM Standard C769-09 "Standard Test Method for Sonic Velocity in Manufactured Carbon and Graphite Materials for Use in Obtaining Young's Modulus" ASTM International, West Conshohocken, PA (2009).

10. ASTM Standard C559-90 "Standard Test Method for Bulk Density by Physical Measurements of Mnaufactured Carbon and Graphite Articles" ASTM International, West Conshohocken, PA (2010).

11. ASTM Standard D7219-08 "Standard Specification for Isotropic and Near-Isotropic Nuclear Graphites” ASTM International, West Conshohocken, PA (2008). 